AJ, 2008 MARCH, IN PRESS

Preprint typeset using $\mathrm{IATEX}_{\mathrm{E}} \mathrm{X}$ style emulateapj v. 08/22/09

\title{
MASS OUTFLOW AND CHROMOSPHERIC ACTIVITY OF RED GIANT STARS IN GLOBULAR CLUSTERS
} I: M15

\author{
Sz. Meszaros ${ }^{1,2,3}$, A. K. Dupree ${ }^{1,4}$, And A. Szentgyorgyi ${ }^{1,5}$ \\ AJ, 2008 March, in press
}

\begin{abstract}
High resolution spectra of 110 selected red giant stars in the globular cluster M15 (NGC 7078) were obtained with Hectochelle at the MMT telescope in 2005 May, 2006 May, and 2006 October. Echelle orders containing $\mathrm{H} \alpha$ and $\mathrm{Ca}$ II $\mathrm{H} \& \mathrm{~K}$ are used to identify emission and line asymmetries characterizing motions in the extended atmospheres. Emission in $\mathrm{H} \alpha$ is detected to a luminosity of $\log \left(L / L_{\odot}\right)=2.36$, in this very metal deficient cluster, comparable to other studies, suggesting that appearance of emission wings is independent of stellar metallicity. The faintest stars showing $\mathrm{H} \alpha$ emission appear to lie on the asymptotic giant branch (AGB) in M15. A line-bisector technique for $\mathrm{H} \alpha$ reveals outflowing velocities in all stars brighter than $\log \left(L / L_{\odot}\right)=2.5$, and this outflow velocity increases with stellar luminosity, indicating the mass outflow increases smoothly with luminosity. Many stars lying low on the AGB show exceptionally high outflow velocities (up to $10-15 \mathrm{~km} \mathrm{~s}^{-1}$ ) and more velocity variability (up to $6-8 \mathrm{~km} \mathrm{~s}^{-1}$ ), than red giant branch (RGB) stars of similar apparent magnitude. High velocities in M15 may be related to the low cluster metallicity. Dusty stars identified from Spitzer Space Telescope infrared photometry as AGB stars are confirmed as cluster members by radial velocity measurements, yet their $\mathrm{H} \alpha$ profiles are similar to those of RGB stars without dust. If substantial mass loss creates the circumstellar shell responsible for infrared emission, such mass loss must be episodic.
\end{abstract}

Subject headings: stars: chromospheres - stars: mass loss - stars: AGB and post-AGB - globular clusters: general - globular clusters: individual (M15)

\section{INTRODUCTION}

The well-known second parameter problem in globular clusters (Sandage \& Wildev 1967), in which a parameter other than metallicity, affects the morphology of the horizontal branch, remains unresolved. Metallicity, as first noted by Sandage \& Wallerstein (1960), remains the principal parameter, but pairs of clusters, with the same metallicity, display quite different horizontal branch morphologies thus challenging the canonical models of stellar evolution and leading to the need for a 'Second Parameter'. Cluster ages have been examined in many studies (Searle \& Zinn 1978; : Lee et al.|1994; Stetson et al.|1996; Lee \& Carnev 1999; Sarajedini 1997; Sarajedini et al. 1997) and in addition, many other suggestions for the 'second parameter(s)' have been proposed, including: total cluster mass; stellar environment (and possibly free-floating planets); primordial He abundance; postmixing surface helium abundance; $\mathrm{CNO}$ abundance; stellar rotation; and mass loss (Catelan 2000; Catelan et al 2001; Sills \& Pinsonneault 2000; Soker et al. 2001; Sweigart 1997; Buonanno et al. 1993; Peterson et al. 1995; Buonanno et al. 1998; Recio-Blanco et al. 2006). Many authors (Vandenberg et al. 1990; Lee et al. 1994; Catelan 2000) have proposed that more than one second parameter may exist in addition to age. One parameter may be mass loss which, as Catelan (2000) notes, remains

\footnotetext{
${ }^{1}$ Harvard-Smithsonian Center for Astrophysics, Cambridge, MA 02138

2 Department of Optics and Quantum Electronics, University of Szeged, 6701 Szeged, Hungary

3 e-mail address: meszi@cfa.harvard.edu

4 e-mail address: adupree@cfa.harvard.edu

${ }^{5}$ e-mail address: saint@cfa.harvard.edu
}

an 'untested second-parameter candidate'.

This spectroscopic study addresses the presence of mass loss from luminous stars in the globular cluster M15. Subsequent papers will include other clusters. Although stellar evolution theory predicts that low-mass Population II stars ascending the red giant branch (RGB) for the first time must lose mass (Renzini 1981; Sweigart et al. 1990), few observations have identified the ongoing mass loss process. Evidence from the period-luminosity relation for RR Lyrae stars suggests that the luminosity variations can be accommodated theoretically if mass loss $\sim 0.2-0.4 M_{\odot}$ has occurred (Fusi Pecci et al. 1993; Christy 1966). Circumstellar CO emission in M-type irregular and semiregular asymptotic giant branch (AGB)-variables implies mass loss rates on the AGB $\sim 10^{-7}-10^{-8} M_{\odot} y r^{-1}$ (Olofsson et al. 2002). Indirect evidence of mass loss processes would be detection of an intracluster medium. These efforts have been marginally successful. Diffuse gas $\left(<1 M_{\odot}\right)$ was suggested in NGC 2808 through the detection of $21-\mathrm{cm} \mathrm{H}$ line emission (Faulkner et al. 1991), but has remained unconfirmed. Ionized intracluster gas was found in the globular cluster 47 Tucanae by measuring the radio dispersion of millisecond pulsars in the cluster (Freire et al. 2001). The central electron density was derived $\left(n_{e}=0.067 \pm 0.0015 \mathrm{~cm}^{-3}\right)$ and found to be two orders of magnitude higher than the ISM in the vicinity of 47 Tuc (Tavlor \& Cordes 1993). Freire et al. (2001) determined the electron density in M15 using four millisecond pulsars to be higher $\left(n_{e} \sim 0.2 \mathrm{~cm}^{-3}\right)$ than in 47 Tuc.

Indirect evidence of mass loss processes comes also from infrared observations. Origlia et al. (2002) using 
ISOCAM images found a mid-IR excess associated with giants in several globular clusters and attributed to dusty circumstellar envelopes. The first detection of intracluster dust in M15 was made by Evans et al. (2003) from the analysis of far infrared imaging data obtained with the ISO instrument ISOPHOT. van Loon et al. (2006) also presented a tentative detection of $0.3 M_{\odot}$ of neutral hydrogen in M15. Smith et al. (1995) placed an upper limit of $0.4 M_{\odot}$ for the molecular gas in M15 from CO observations with the 15-m James Clerk Maxwell Telescope on Mauna Kea. Using the Spitzer Space Telescope, Boyer et al. (2006) detected a population of dusty red giants near the center of M15. Observations from Spitzer with the Multiband Imaging Photometer for Spitzer (MIPS) also revealed the intracluster medium (ICM) discovered by Evans et al. (2003) near the core of the globular cluster. As Origlia et al. (2002) noted, the infrared detections may only be tracing the outflowing gas and may not be related to the driving mechanisms for the wind. More recently Origlia et al. (2007) identified dusty RGB stars in 47 Tuc and derived an empirical mass loss law for Population II stars. Mass loss rates derived from these observations showed that the mass loss increases with luminosity and possibly episodic.

High resolution stellar spectroscopy allows the direct detection of mass outflow from the red giants themselves. Emission in the wings of $\mathrm{H} \alpha$ lines in the spectra of globular cluster red giants was first described in detail by Cohen (1976). Later observations revealed that emission in $\mathrm{H} \alpha$ is common in globular clusters and night-to-night variations can occur (Mallia \& Pagel 1981; Peterson 1981, 1982; Cacciari \& Freeman 1983; Gratton et al. 1984). These studies have shown that most of the stars brighter than $\log \left(L / L_{\odot}\right) \approx 2.7$ exhibit $\mathrm{H} \alpha$ emission wings. The emission itself is likely not a direct indicator of mass loss, because emission can arise from an optically thick stellar chromosphere surrounding the star (Dupree et al. 1984). Variation of the strength of emission can also be affected by stellar pulsation (Smith \& Dupree 1988). Better mass flow indicators in the optical are the line coreshifts or asymmetries of the $\mathrm{H} \alpha$ or Ca II H\&K profiles and emission features. Red giants in globular clusters (M22 and Omega Centauri) were found to have velocity shifts less than $14 \mathrm{~km} \mathrm{~s}^{-1}$ in the cores of $\mathrm{H} \alpha$ relative to the photospheric lines (Bates et al. 1990, 1993). These results were similar to metal-poor field giants, where only giants brighter than $M_{V}=-1.7$ have emission wings and the line shifts were $<9 \mathrm{~km} \mathrm{~s}^{-1}$ (Smith \& Dupree 1988) indicating very slow outflows and inflows in the chromosphere. For globular clusters, Lyons et al. (1996) discussed the $\mathrm{H} \alpha$ and $\mathrm{Na} I \mathrm{D}$ line profiles for a sample of 63 RGB stars in M4, M13, M22, M55, and $\omega$ Cen. The coreshifts were less than $10 \mathrm{~km} \mathrm{~s}^{-1}$, much smaller than the escape velocity from the stellar atmosphere at $2 R_{*}\left(\approx 50-70 \mathrm{~km} \mathrm{~s}^{-1}\right)$. Dupree et al. (1994) studied 2 RGB stars in NGC 6752 and found that the Ca II $\mathrm{K}$ and $\mathrm{H} \alpha$ coreshifts were also low (less than $10 \mathrm{~km} \mathrm{~s}^{-1}$ ). However, asymmetries in the Mg II lines showed a stellar wind with a velocity of $\approx 150 \mathrm{~km} \mathrm{~s}^{-1}$, indicative of a strong outflow. Mg II lines are formed higher in the atmosphere than $\mathrm{H} \alpha$ and Ca II K, which suggests that the stellar wind becomes detectable near the top of the chromosphere. A detailed study was carried out by Cacciari et al. (2004), who observed 137 red giant stars in NGC 2808. Most of the stars brighter than $\log \left(L / L_{\odot}\right)=2.5$ clearly showed emission wings in $\mathrm{H} \alpha$. The velocity shift of the $\mathrm{H} \alpha$ line core compared to the photosphere is less than $\approx 9 \mathrm{~km} \mathrm{~s}^{-1}$. Outward motions were also found in both $\mathrm{Na}$ I D and Ca II K profiles.

This paper discusses high-resolution spectroscopy of the $\mathrm{H} \alpha$ and $\mathrm{Ca}$ II H\&K lines of red giant stars in M15. Our deep sample of M15 giants in this metal poor cluster $([\mathrm{Fe} / \mathrm{H}]=-2.26)$ offers a good comparison to other studies of more metal rich clusters. Also, the high radial velocity of M15 $\left(-105 \mathrm{~km} \mathrm{~s}^{-1}\right)$ minimizes contamination by interstellar absorption in the profiles of resonance lines.

\section{OBSERVATIONS AND DATA REDUCTION}

Observations of a total of 110 red giant stars in M15 were made in 2005 May, 2006 May, and 2006 October with Hectochelle on the MMT (Szentgyorgyi et al. 1998). Hectochelle uses 240 fibers each of which subtends $\sim 1.5$ arcsec in the sky. Hectochelle covers 1 degree of sky, yet the apparent diameter of M15 is only 10 arc minutes, so that about 50-60 red giants could be measured in M15 for each configuration. The requirement that fibers can not be placed closer than 2 arcsec apart further constrains the target selection. In addition, we wanted to search for variability which led to multiple visits for many targets over the 17 month span. Software (xfitfibs) has been developed at CfA to optimize the fiber configuration with specified priorities and requirements.

Targets were chosen from the catalog of Cudworth $(1976)$ to have a high probability $(>95 \%)$ of membership and to provide smooth coverage of the RGB and AGB within the constraint of the fiber placement on the sky. The color magnitude diagram (CMD) of the observed cluster members can be seen in Figure 1 and they are listed in Table 1. Coordinates of the stars were taken from the 2MASS catalog (Skrutskie et al. 2006) and used to position the fibers. Additional targets from Cudworth's list with lower membership probability and field targets from the 2MASS catalog were included. Many fibers were placed on the blank regions of the sky in order to measure the sky background in detail. The sky fibers were equally distributed in the observed field to cover a large area around the cluster. Since Hectochelle is a single-order instrument, two orders were selected with order-separating filters: $\mathrm{H} \alpha$ (region used for analysis $\lambda \lambda 6475-6630)^{6}$, and Ca II H\&K (region used for analysis $\lambda \lambda 3910-3990)$ to give $155 \AA$ centered on the principal spectral features in $\mathrm{H} \alpha$ and $80 \AA$ in $\mathrm{Ca}$ II $\mathrm{H} \& \mathrm{~K}$. The spectral resolution was about 34,000 as measured by the FWHM of the ThAr emission lines in the comparison lamp. Exposures in each of the two orders are summarized in Table 2.

Data reduction was done using standard IRAF spectroscopic packages. The IRAF package ccdproc performed the trimming and the overscan correction and made the bad pixel mask. The average bias image was subtracted from every spectrum. Correcting with the dark images was not necessary because even in the 40 minute dark exposures the intensity was very low $[3-4$ analog digital

6 The 2005 May observation had less wavelength coverage. See following text. 
units (ADU) per pixel]. To find and trace the apertures, ten flat images were taken with the continuum lamp of 10 seconds exposure time each. The focal plane of Hectochelle consists of a mosaic of 2 CCDs that are slightly misaligned. The aperture finding algorithm fails near the crack between the two CCDs, so manually editing the apertures and reordering them was necessary. Some apertures were deleted from the edges of the CCD and a total of 240 orders was extracted. To correct for the pixel-to-pixel variations, the averaged continuum flat exposures for each configuration were fitted with a 21st order spline function (using the IRAF task apflatten) and used to divide the corresponding object spectra by the normalized flat. A region 13-pixels wide was used for the aperture extraction. Wavelength standards, using Thorium-Argon (ThAr) hollow cathode lamps were taken to define the wavelength scale; each ThAr image had 900 seconds of exposure time and was taken at the beginning of the night. We identified $15-20$ strong ThAr emission lines in the first aperture, then propagated these identifications to every other aperture manually to check the accuracy of the fit. During the calibration the rms of the wavelength fit had to be between $0.01-0.002 \AA$ to reach the theoretical resolution of the spectrograph. If the error of the fit is larger than this, it will be comparable to the expected width of the ThAr features $(0.1-0.2 \AA)$ and increase the error of the wavelength solution. The continuum flat images were used to correct the throughput for each aperture using a region close to the CCD center containing $5-7$ neighboring fibers. An average of the selected continuum flat apertures was taken and divided into all other apertures in the same exposure and was used to correct the vignetting and fiber-to-fiber throughput deviation.

The extracted spectra also contain sky background which had to be subtracted. Some of the sky apertures showed weak $\mathrm{H} \alpha$ and other photospheric lines suggesting very faint stars in those positions. Also very bright stars can cause scattered light in the neighboring apertures on the CCD, but this becomes visible only if the aperture has more than $8000-10000$ ADUs per pixel. The brightest stars reached 10000 ADUs per pixel in the 40 minute exposures and some apertures contained very low level scattered light. Every sky aperture was checked carefully and those where faint stars or scattered light were found were discarded. A median filtered sky was used for the subtraction, but sky subtracted skies frequently contained additional counts, which changed aperture by aperture and by wavelength. In Figure 2, sky intensity versus wavelength and aperture is plotted. Between aperture numbers 100 and 150, especially at longer wavelengths, the apertures have higher intensity. The dark images did not show high intensity features and the intensity pattern in Figure 2 is currently not understood. To subtract the sky, the images were divided into 3 different aperture sections, and the sky subtraction was done with one of two methods. In the first and third segments (corresponding to aperture numbers $0-100$ and $150-240$ ), the intensity appears constant as a function of aperture number and wavelength so the median filtered spectrum could be used for subtraction. The middle region spanned aperture numbers 101 to 149 . In this region the sky was subtracted from every target aperture using the average of 3 closest sky apertures on the CCD itself.

In the 2005 May spectra of $\mathrm{H} \alpha$, the filter's central wavelength was offset by $\sim 80 \AA$ placing the $\mathrm{H} \alpha$ line near the long wavelength end of the CCD. Fluxes at wavelengths shorter than $\mathrm{H} \alpha$ were abnormally low, because the grating was so far off the blaze angle. The wavelength regions spanned by the OB25 filter differed between the 2005 and 2006 observations; however both contained the $\mathrm{H} \alpha$ line and photospheric lines.

\section{STARS WITH H $\alpha$ EMISSION, RADIAL VELOCITIES}

\subsection{The Color Magnitude Diagram and Physical Parameters}

We observed a total of 110 different red giant stars in M15 and found 29 with $\mathrm{H} \alpha$ emission. About half of them were observed more than once. Emission above the continuum in the $\mathrm{H} \alpha$ profile can be seen in Figures $3-6$. For comparison each figure includes a star that exhibits no emission. The color-magnitude diagram (CMD) for each night of observation appears in Figure 7. On 2005 May 22, emission is found in stars of $\mathrm{V}=14.48$ and brighter, corresponding to $M_{V}=-0.89$, using the apparent distance modulus $(m-M)_{V}=15.37$ from Harris (1996). Stars in M15 that show emission occurred at different magnitude limits on different dates of observation $\left(M_{V}=-1.17\right.$ on 2006 May $11, M_{V}=-0.99$ on 2006 October 4 and $M_{V}=-1.68$ on 2006 October 7). Studies of metal deficient field giants found emission in objects brighter than $M_{V}=-1.7$ (Smith \& Dupree 1988), whereas in the metal rich cluster NGC 2808 the detection threshold for emission was set at $M_{V}=-1.0$ (Cacciari et al. 2004). It is well documented that the presence of $\mathrm{H} \alpha$ varies with time, and this appears to be the most likely explanation of the differences in the detection level of the $\mathrm{H} \alpha$ emission.

For comparison on a luminosity scale, unreddened colors for M15 stars were calculated taking $E(B-V)=0.10$, $E(V-K)=2.75 E(B-V)$, and apparent distance modulus $(m-M)_{V}=15.37$ Cardelli et al. 1989; Harris 1996). The effective temperatures, bolometric corrections, and luminosities were obtained from the $V-K$ visual colors (Table 3 ) using the empirical calibrations by Alonso et al. (1999, 2001) and the cluster average metallicity $[\mathrm{Fe} / \mathrm{H}]=-2.26$ (Harris 1996).

Stars brighter than $\log \left(L / L_{\odot}\right)=2.36$ can exhibit emission, and all together $\sim 46 \%$ of these show $\mathrm{H} \alpha$ emission. The asymmetry of the $\mathrm{H} \alpha$ emission wings was noted for emission above the continuum level where B represents the strength of the short wavelength (blue) emission and $\mathrm{R}$ denotes the strength of the long wavelength (red) emission. Figure 7 shows the CMD for our targets where the asymmetry of the $\mathrm{H} \alpha$ line is indicated. The frequency of $\mathrm{H} \alpha$ emission increases with the stellar luminosity, however the line asymmetry is not correlated with color and luminosity. Stars with $\mathrm{B}<\mathrm{R}$ and $\mathrm{B}>\mathrm{R}$ seem equally distributed in luminosity. Among stars with emission, the majority $(\sim 75 \%)$ exhibit emission wings with $\mathrm{B}>\mathrm{R}$, a signature generally considered to indicate inflow of material.

Spectra of 29 stars with $\mathrm{H} \alpha$ emission were obtained in both 2005 and 2006. All but two of these stars showed significant changes in the line emission which either ap- 
peared, or vanished, or changed asymmetry (see Table 4 , and Figures 3-6).

\subsection{Radial Velocities}

To measure accurate radial velocities we chose the cross-correlation method using the IRAF task xcsao. Using the ATLAS (Kurucz 1993) code, we synthesized the spectrum of a red giant star, K341, in M15. This is a bright star with a high quality spectrum, thus the comparison between the observed and modeled spectrum is optimum. The physical parameters of our template spectrum were the following: $T_{\text {eff }}=$ $4275 \mathrm{~K}, \log g=0.45, v_{\text {turb }}=0 \mathrm{~km} \mathrm{~s}^{-1}, v_{\text {macro }}=0$ $\mathrm{km} \mathrm{s}^{-1},[\mathrm{Fe} / \mathrm{H}]=-2.45,[\mathrm{Na} / \mathrm{Fe}]=0.01,[\mathrm{Si} / \mathrm{Fe}]=0.4$, $[\mathrm{Ca} / \mathrm{Fe}]=0.56,[\mathrm{Ti} / \mathrm{Fe}]=0.57,[\mathrm{Ba} / \mathrm{Fe}]=0.2($ Sneden et al . 2000). This spectrum is computed in LTE and a chromosphere was not included in the atmospheric model. The comparison between the template in our cross-correlation and the observed Hectochelle spectrum can be seen in Figure 8. The region selected for the cross-correlation spanned $6480 \AA$ to $6545 \AA$ purposely omitting the $\mathrm{H} \alpha$ line. The telluric and photospheric lines were identified using the synthesized spectrum of K341. In this region there are many telluric lines of water vapor. These lines appear in the cross-correlation function profile as an additional peak, well separated from the cluster velocity, and so the measured stellar radial velocity is not affected. To verify our radial velocities, we cross-correlated a narrow region $\lambda \lambda 6480-6500$ where no strong telluric lines are found. Cross-correlating this narrow region results in the same radial velocity as from the broader window, but with a larger $\left(1-2 \mathrm{~km} \mathrm{~s}^{-1}\right)$ error.

The $\mathrm{H} \alpha$ spectra of our targets were also crosscorrelated against several hundred spectra calculated by Coelho et al. (2005) covering temperatures between 3500 and $7000 \mathrm{~K}$ and metallicities between $[\mathrm{Fe} / \mathrm{H}]=-2.5$ and +0.5 . These velocities from the Coelho spectra agreed within 1 to $2 \mathrm{~km} \mathrm{~s}^{-1}$ with our earlier determination using only the K341 template, because the same photospheric Fe and Ti absorption lines can be found in all the spectra.

There is good agreement between our radial velocities from the 2005 data and those of Gebhardt et al. (1997) and Peterson et al. (1989) (see Figure 9, top panels). Gebhardt et al. (1997) used an Imaging FabryPerot Spectrophotometer with the Sub-arcsecond Imaging Spectrograph on the Canada-France-Hawaii Telescope and observed 1534 stars in M15 with velocity errors between 0.5 and $10 \mathrm{~km} \mathrm{~s}^{-1}$. Peterson et al. (1989) used echelle spectrographs on the MMT, the $1.5 \mathrm{~m}$ Tillinghast reflector of the Whipple Observatory on Mount Hopkins, and the 4-m telescope of Kitt Peak National Observatory. Peterson et al. (1989) quote an average error of $1 \mathrm{~km} \mathrm{~s}^{-1}$, but the stars in common with our sample have larger errors $\left(1-2 \mathrm{~km} \mathrm{~s}^{-1}\right)$. However the Hectochelle velocities from 2006 display a systematic offset from the 2005 measurements of the same stars (see Figure 9, lower panels). This offset amounts to $+1.9 \pm 0.5 \mathrm{~km} \mathrm{~s}^{-1}$ and $+0.9 \pm 0.5 \mathrm{~km} \mathrm{~s}^{-1}$ for 2006 May and Oct 2006 respectively, and the data in Table 5 were corrected for this systematic offset. The radial velocities of the sky emission lines show the same effect. In 2005, all of the sky emission lines were at $0 \mathrm{~km} \mathrm{~s}^{-1}$ and in 2006 May were at $-2 \mathrm{~km} \mathrm{~s}^{-1}$, yet the wavelength calibration of the 2006 data appears to be as accurate as 2005 . The source of this offset comes from revisions made to the calibration system of Hectochelle that changed the illumination in the spectrograph. The amount of the offset is small, and does not affect determination of cluster membership. The average cluster radial velocity was calculated using velocity-corrected data from all four observations. Our value is $-105.0 \pm 0.5 \mathrm{~km} \mathrm{~s}^{-1}$, which is slightly lower than the cluster radial velocity $\left(-107.0 \pm 0.2 \mathrm{~km} \mathrm{~s}^{-1}\right)$ quoted in the Harris (1996) catalog.

Previous studies suggested that several of our sample stars are binaries. Significant velocity variations, $\approx 6.5 \mathrm{~km} \mathrm{~s}^{-1}$, for K47 were found by Soderberg et al. (1999), but our measurements showed only $0.9 \mathrm{~km} \mathrm{~s}^{-1}$ variation between 2005 May and 2006 May. This change lies within the measurement errors $\left(\approx 1 \mathrm{~km} \mathrm{~s}^{-1}\right)$. K757 and K825 were suggested as binaries by Sneden et al. (1997) from the asymmetric line profiles; weak satellite wings were visible for nearly all spectral lines. We have only one observation of K825, but K757 changed by $6.2 \mathrm{~km} \mathrm{~s}^{-1}$, which could indicate that this star is a binary. Drukier et al. (1998) found 17 cluster members of M15 showing possible radial velocity variability. Four of these stars were observed with Hectochelle, but only one of them, K92, was observed more than once. This star showed $1.4 \mathrm{~km} \mathrm{~s}^{-1}$ variability between 2005 May 22 and 2006 October 7, but the error of these observations was close to $1 \mathrm{~km} \mathrm{~s}^{-1}$. Six additional stars showed velocity changes larger than $2 \mathrm{~km} \mathrm{~s}^{-1}$, which could indicate these stars are binaries: B5 $\left(6.8 \mathrm{~km} \mathrm{~s}^{-1}\right)$, B30 $\left(2.9 \mathrm{~km} \mathrm{~s}^{-1}\right), \mathrm{K} 757\left(6.2 \mathrm{~km} \mathrm{~s}^{-1}\right), \mathrm{K} 1084\left(2.6 \mathrm{~km} \mathrm{~s}^{-1}\right)$, $\mathrm{K} 1097\left(2.1 \mathrm{~km} \mathrm{~s}^{-1}\right)$, and K1136 $\left(3.0 \mathrm{~km} \mathrm{~s}^{-1}\right)$.

Some of the M15 targets selected from the proper motion study of M15 (Cudworth 1976) turned out to have substantially different radial velocities from the cluster average (see Table 6) and are not likely to be members of the cluster. These stars are not included in the spectroscopic analysis.

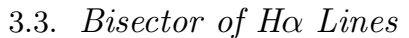

The core of the $\mathrm{H} \alpha$ line is formed higher in the stellar chromosphere than the line wings and is expected to give an indication of the atmosphere dynamics. We first measured the position of the $\mathrm{H} \alpha$ absorption line core relative to photospheric lines using the IRAF task splot. We found an error in the wavelength scales depending on the aperture number that prevented measurement of the core offset of $\mathrm{H} \alpha$ to better than $\pm 2 \mathrm{~km} \mathrm{~s}^{-1}$. At present, we believe that the different light path of the ThAr comparison lamp from that of the stellar spectra causes this error, which appeared as a variable 'stretching' of the wavelength scale dependent on aperture and zenith distance of $\mathrm{M} 15^{7}$.

Thus, a better approach to the velocity differences consists of measuring the line asymmetry using a line bisector. The difference between the centers of the line core and of the line near the continuum level gives a measure of the atmospheric dynamics through the chromosphere. To accomplish this, the line profile was divided into 20 sectors in normalized flux. The top sector was usually

\footnotetext{
7 Since this discovery, new observational procedures have been instituted with Hectochelle using sky spectra to eliminate these effects.
} 
between 0.7 and 1.0 of the continuum in the normalized spectrum, the lowest sector was placed $0.01-0.05$ above the lowest value of the line depending on its signal-tonoise ratio. The velocities of the $\mathrm{H} \alpha$ bisector asymmetry $\left(v_{b i s}\right)$ are calculated in the following way: the top and the bottom 3 sectors are selected, the wavelength average of each sector is calculated, then subtracted one from another and changed to a velocity scale. The bisector velocities, $v_{b i s}$, are shown in Figure 10 and listed in Table 7. A negative value corresponds to an outflowing velocity. Probable errors for these measurements were formally calculated and range from $0.5 \mathrm{~km} \mathrm{~s}^{-1}$ to $1.5 \mathrm{~km} \mathrm{~s}^{-1}$ for the brightest to faintest stars respectively. Stars fainter than $\log \left(L / L_{\odot}\right)=2.5(\mathrm{~V} \approx 14.20)$ did not show $\mathrm{H} \alpha$ asymmetry and $v_{b i s}$ was nearly zero. Stars brighter than $\log \left(L / L_{\odot}\right)=2.5(\mathrm{~V} \approx 14.20)$ showed asymmetry and in almost every case the line profile was blue shifted. This magnitude limit is comparable to the luminosity limit of stars showing emission, however stars with a blue shifted $\mathrm{H} \alpha$ core did not always show emission wings. The core velocity evidently is a more sensitive diagnostic of outflows. The amplitude of $v_{b i s}$ correlates with the luminosity (Figure 10). Where AGB stars are well separated in color from the RGB stars, near $\log L / L_{\odot}=2.3$ to 2.6, the AGB stars exhibit larger values of $v_{b i s}$ and more variability than RGB stars (Figure 11). At higher luminosities, where the AGB and RGB objects can not be distinguished from one another in the CMD, the bisector velocities are all significantly higher than stars at fainter magnitudes on the RGB.

\subsection{Ca II K Profiles}

Spectra of 53 red giant stars in the Ca II H\&K region were obtained in 2005 and the profiles of the $\mathrm{Ca} \mathrm{K}$ core $(\lambda 3933)$ are shown in Figures 12 and 13 for most stars showing emission. Two other stars, K702 and K1029, not shown in these figures, exhibit Ca emission too. The spectra had low signal to noise ratio $(\mathrm{S} / \mathrm{N} \approx 15-20$ in the continuum and $\approx 5-10$ in the core) and it is difficult to identify emissions in many cases. Continuum normalization is challenging in this spectral region because spectral synthesis demonstrates that hundreds of absorbing lines generally depress the continuum substantially. A low order Chebyschev function was used to fit and normalize the local continuum away from the strong Ca II lines. In many stars, the noise may be comparable to the emission in the core of the $\mathrm{K}$ line, preventing measurement of the radial velocity of the central self-absorption in almost all spectra. The presence of emission is determined by eye by comparison to the synthesized spectrum of K341 constructed with the Kurucz code (Kurucz 1993) using the physical parameters described in Section 3.2. This model spectrum contains only photospheric lines and no chromosphere was included in the model, thus making it excellent for detection of emission. It is clear that the stellar spectrum in the line core does not reach zero flux as would be expected in such a deep photospheric line. Some additional counts in the core may come from the inaccurate sky subtraction. We found fourteen stars out of 53 stars where Ca II $\mathrm{K}$ emission is definite or highly likely. Eleven of these stars showed $\mathrm{H} \alpha$ emission when measured one day earlier in 2005. In the 11 stars where the Ca asymmetry is obvious, the ratio of the blue to the red side of the $\mathrm{Ca} \mathrm{K}$ emission core (the core asymmetry) could be assessed (see Table 8) and in 6 out of the 11 cases, the core asymmetry differed from the asymmetry as noted in $\mathrm{H} \alpha$.

\section{DISCUSSION}

\subsection{The Ho line}

One hundred and ten stars were observed during 2005 and 2006 and twenty-nine of these had $\mathrm{H} \alpha$ emissions. The magnitude of the faintest star showing $\mathrm{H} \alpha$ emission above the continuum varied among our 4 observations. This is not surprising based on the variability in the presence, strength, and emission asymmetries found by us and by others (Cacciari \& Freeman 1983). The spectra of 2005 May 22 reveal emission in the faintest giant, $\mathrm{K} 582$, at $\log \left(L / L_{\odot}\right)=2.36(\mathrm{~V}=14.48)$. This star and several others (K158, K260, K482, K875, K979) displaying emission may be located on the AGB as judged by the CMD (Figure 7) where they are distinct from the red giant branch. Stars brighter than $\mathrm{V} \sim 14$ can not be clearly separated into AGB and RGB from the colormagnitude diagram alone.

Inspection of the bisector velocities reveals differences among the red giant stars. None of the stars brighter than $\mathrm{V} \sim 14.5, \log \left(L / L_{\odot}\right)>2.4-2.5$ have red-shifted $\mathrm{H} \alpha$ cores (Figure 10); all are blue-shifted or exhibit no shift at all. This luminosity cutoff corresponds also to the limit of the $\mathrm{H} \alpha$ emission wings which suggests that the blue-shift and the emission are related. The emission wings arise deep in the chromosphere as models have shown (Dupree et al. 1984; Mauas et al. 2006), and the dynamics of the upper chromosphere are reflected in the line core. Motions start in the lower atmospheric layers and then progress outward through the chromosphere. The amount of the bisector shift increases with stellar luminosity (Figure 10).

There are several stars that show faster outflow asymmetries than the generally low-speed outflows near $\log L / L_{\odot}=2.3$ to 2.6 (Figure 10). These stars, K158, K260, K582, K875, and K979 display bisector velocities ranging from $-6.7 \mathrm{~km} \mathrm{~s}^{-1}$ to $-13.2 \mathrm{~km} \mathrm{~s}^{-1}$ in comparison to the remainder of stars in that magnitude interval where bisector velocities are typically less than $-5 \mathrm{~km} \mathrm{~s}^{-1}$. One of these stars, K260 showed the largest change in core-shift velocity measuring $-3.0 \pm 1.2 \mathrm{~km} \mathrm{~s}^{-1}$ in 2005 and $-10.6 \pm 0.9 \mathrm{~km} \mathrm{~s}^{-1}$ in 2006 . The position of the high-outflow stars in the CMD suggests they are AGB stars since they lie blueward of the fiducial AGB in Figure 7. These objects appear to be AGB stars, and the relatively high outflows mark the presence of a substantial stellar wind. However, as Figure 11 shows, other stars near the fiducial AGB do not have high outflow velocities. If these low velocity objects are also AGB stars, then this argues for an episodic outflow.

The asymmetry of the $\mathrm{H} \alpha$ wings indicates that most of the giant stars have inflow motions in the region where the wings are formed. Since it appears likely that these stars are pulsating (Mayor et al. 1984), although this may be controversial for the metal deficient field giants (Carnev et al. 2003), it is of interest to compare the asymmetry pattern with that of Cepheids which are known pulsators. One well-studied Cepheid, $\ell$ Car (HD 84810), shows variable emission wings in $\mathrm{H} \alpha$ similar to those found here (Baldry at al. 1997). The appearance 
of $B / R<1$ asymmetry generally coincides with blueshifted photospheric metal absorption lines; and the converse applies, when $B / R>1$, the photospheric lines are red-shifted. So a dynamic linking clearly exists between the photosphere and the regions forming the $\mathrm{H} \alpha$ line wings. The radial velocity of this long-period (35 days) Cepheid shows photospheric red-shifts for about half of its pulsation period, and 'inflow' $\mathrm{H} \alpha$ line asymmetries for about 0.35 of its period. Inspection of radial velocity curves from metallic lines shows that longer-period Cepheids have red shifts for a greater proportion of their pulsation period (Nardetto et al. 2006; Petterson et al. 2005). Thus it is not surprising that the $\mathrm{H} \alpha$ profiles in the red giants in M15 show a dominant inflow asymmetry.

Models for dust-free Mira stars (Struck et al. 2004) indicate that lower levels of the atmosphere can support radial pulsations which develop into a steady outflow at larger distances. A similar behavior is suggested by the outflows detected in the cores of the $\mathrm{H} \alpha$ lines for the M15 giants. Models of the $\mathrm{H} \alpha$ line profiles for metal deficient giants show that the core is formed at a mass column density substantially above the region forming the wings (Dupree et al. 1984; Mauas et al. 2006). Such models for red giants are needed to explore the dynamics of the atmospheres and to evaluate the mass loss rate.

\subsection{Ca II Emission}

Fourteen of the red giants showed emission in Ca II K and 12 spectra are shown in Figures 12 and 13 . The lower luminosity limit is at least the same as found for the presence of $\mathrm{H} \alpha$ emission, namely $\log \left(L / L_{\odot}\right)=2.36$. The luminosity limits of Ca II $K_{2}$ emission and $\mathrm{H} \alpha$ appear to be related. Calcium emission may well extend to fainter limits; our spectra of fainter stars did not have sufficient signal to identify emission in the center of the deep photospheric Ca II line. However, three of the stars displaying $\mathrm{Ca}$ II $\mathrm{K}$ emission do not have $\mathrm{H} \alpha$ emission, but this is not suprising since the presence of $\mathrm{H} \alpha$ emission is known to vary (see Table 4 ). While the spectra are noisy, the $\mathrm{Ca}$ II $\mathrm{K}$ asymmetries seem to include all possibilities: $B<R, B=R, B>R$. These asymmetries differ in 6 stars from the asymmetries of the $\mathrm{H} \alpha$ wing in each star. Such a difference is not unexpected since the regions of formation of the $\mathrm{Ca} \mathrm{K}$ core and the $\mathrm{H} \alpha$ wings are separated in the atmosphere of a giant $\left(\mathrm{Ca} \mathrm{K}_{2}\right.$ emission forms higher in the atmosphere than $\mathrm{H} \alpha$ wings); additionally $\mathrm{H} \alpha$ shows variations in asymmetries over a few days (Cacciari \& Freeman 1983) which could contribute to the differences.

\subsection{Comparison with Other Studies}

A detailed spectroscopic study (Cacciari et al. 2004) was made of 137 RGB stars in NGC 2808 which extended to $\sim 3$ magnitudes below the tip of the red giant branch. The majority of their targets were at lower resolution than we have here, however 20 were sampled at high resolution. Emission in $\mathrm{H} \alpha$ was detected down to a limit of $\log \left(L / L_{\odot}\right)=2.5$ which is about 0.15 magnitude brighter than we find in M15. NGC 2808 is more metal rich $([\mathrm{Fe} / \mathrm{H}]=-1.15$, Harris $(1996))$ than M15 $([\mathrm{Fe} / \mathrm{H}]=-2.26$, Harris $(1996))$ which may account for the slight difference. On the other hand, the distance modulus for M15 was recently determined to be
$15.53 \pm 0.21$ using the zero-age horizontal branch level as a distance indicator (Cho \& Lee 2007) which would bring the luminosities into closer agreement. In the Cacciari et al. (2004) spectra taken at highest resolution, the red giants in NGC 2808 have wing emission in $\mathrm{H} \alpha$ indicative of inflow $(B / R>1)$ in the majority of stars, similar to our results. Other surveys generally contained only the brightest stars in the clusters, and their luminosity limits extend only to $\log \left(L / L_{\odot}\right)=2.7$ (Bates et al. 1993; Lyons et al. 1996). Smith \& Dupree (1988) noted $\mathrm{H} \alpha$ emissions in metal-poor field giants brighter than $\log \left(L / L_{\odot}\right)=2.5$.

While the luminosity limits of the $\mathrm{H} \alpha$ emission are similar in M15 and NGC 2808, the distribution of the emission with luminosity and effective temperatures differs between the clusters. Figure 14 compares the fraction of stars with $\mathrm{H} \alpha$ emission in M15 from this study with NGC 2808 (Cacciari et al. 2004). At the same luminosity, the M15 giants exhibit a lower percentage of $\mathrm{H} \alpha$ emissions than is found in NGC 2808. However, at the same effective temperature, the fraction of stars showing emission is the opposite; fewer stars have emission at the same effective temperature in NGC 2808 than in M15. Although the fraction of stars with emission generally increases with luminosity, the differences result principally because the CMD for NGC 2808 (which is more metal rich than M15) lies at lower effective temperatures at the same luminosity. Assessing the emission fraction as a function of stellar radius (Figure 14, right panel) suggests that the fractions are comparable except at large values of the stellar radius. Possibly the coolest stars can not support the thick chromosphere necessary to produce emission (Dupree et al. 1984; Mauas 2007) and/or the pulsational characteristics of the atmospheres differ.

Cacciari et al. (2004) were able to measure significant core shifts of $\mathrm{H} \alpha$ in 7 stars of their sample of giants in NGC 2808 observed with the high resolution spectrograph UVES (20 stars). Outflow velocities more negative than $-2 \mathrm{~km} \mathrm{~s}^{-1}$ appear for stars brighter than $\log \left(L / L_{\odot}\right)=3.16$ but there is little velocity data for the fainter giants. More stars had measurable velocities in the $\mathrm{Na} \mathrm{D}$ lines, and outflows from $1-4 \mathrm{~km} \mathrm{~s}^{-1}$ became apparent at $\log \left(L / L_{\odot}\right)=3.1$ and brighter. M4, another cluster of similar metallicity as NGC 2808 did not have coreshifts (more negative than $-2 \mathrm{~km} \mathrm{~s}^{-1}$ ) either in $\mathrm{H} \alpha$ or $\mathrm{Na} \mathrm{D}$ in any of $\approx 10$ stars that have luminosities $\log \left(L / L_{\odot}\right)<3.3$ (Kemp \& Bates 1995). By contrast, the velocities in M15 indicated systematic outflow (more negative than $-2 \mathrm{~km} \mathrm{~s}^{-1}$ ) in the $\mathrm{H}$-alpha core occur at lower luminosities, $\log \left(L / L_{\odot}\right)=2.5$. Thus there are possible signs of a metal dependency in the outflow with higher velocities in metal poor objects. More complete sampling of outflows in other clusters is needed.

The luminosity limit (Cacciari et al. 2004) for Ca K emission lines was $\log \left(L / L_{\odot}\right) \sim 2.6$ which is comparable to the $\mathrm{H} \alpha$ limit in NGC 2808 and similar to the results for M15. However our data did not have sufficient signal to noise to check the fainter stars. In sum, while the chromospheric emissions appear independent of metallicity, outflows may be enhanced in low metallicity stars.

\subsection{Spitzer Stars}

Boyer et al. (2006) have observed M15 with the Spitzer Space Telescope using the IRAC and MIPS instruments. 
They concluded that a significant amount of dust $(9 \pm$ $2 \times 10^{-4} M_{\odot}$ ) occurs near the center of the cluster and suggested that this dust comes from the mass-loss of the brightest giant stars. Twenty-three stars were identified as dusty IR sources and their IRAC colors indicate that these are AGB stars. We observed 12 of these stars (Table 1) and could confirm their cluster membership with radial velocity measurements (Table 5 ). We noted one source, designated by Bover et al. (2006) as unidentified (SSTU J212953.33+120910.7) is associated with K272 at the same coordinates. All of the observed Spitzer stars showed blue shifts in $\mathrm{H} \alpha$. Six of them (K224, K421, K479, K672, K709 and GEB254) have strong $\mathrm{H} \alpha$ emission, but only one (K479) had outflow asymmetry in $\mathrm{H} \alpha$ $(\mathrm{B}<\mathrm{R})$. Other stars in the Spitzer field (K144, K260, K393, K431, K447, K462, K702) were not identified as dusty stars, yet their $\mathrm{H} \alpha$ line emissions and blueshifts are similar to those stars identified by Spitzer observations as having an IR excess. Evidently not all stars produce dusty shells. The $\mathrm{H} \alpha$ emission profiles observed here do not seem to be related to a prior phase when the star produced material that cooled down to produce an IR excess.

All twelve Spitzer stars have the same colors and luminosities as other RGB stars on the CMD (Figure 7) and their bisector velocities also show similar values (Figure 10) as other RGB stars at the same luminosity. However the bisector velocity does not appear to correlate with any IRAC colors or magnitudes. The intensity ratio of short wavelength and long wavelength emission peaks $(\mathrm{B} / \mathrm{R})$ and the strength of the $\mathrm{H} \alpha$ emission wings are very similar to other stars in this color and luminosity region of the CMD. In this region, where the Spitzer sources are located, the differences between the AGB stars and RGB stars are hard to discern spectroscopically.

Spitzer showed (Bover et al. 2006) that only some stars in this region of the CMD have a mid-IR excess. Assuming that the Spitzer sources must have had strong stellar winds to produce dust and the current $\mathrm{H} \alpha$ emission profiles are not related to the episode of dust production, one can conclude that the mass loss is not continuous. Origlia et al. (2002) used ISOCAM images to study red giants in globular clusters but the large pixel size of ISOCAM made it difficult to identify stellar sources. Frequently, several stars were candidates for each midIR source in a 15 arcsec square area, and the brightest one was selected. They concluded that strong mass loss occurred only among RGB stars located at the red giant tip $\left[\log \left(L / L_{\odot}\right)>=3\right]$. Not all of the luminous stars identified in this way had in IR excess indicating dust, suggesting that the mass loss was episodic. The results reported here have no ambiguity in identification, and demonstrate the presence of episodic mass loss over a much greater extent in luminosity. These stars must have passed through several active phases with very strong stellar winds during their lifetimes on the AGB.

\section{CONCLUSIONS}

Differences found in the profiles of the $\mathrm{H} \alpha$ line give insight into the atmospheric structure and dynamics. Stars which are physically larger show more emission. At a given luminosity, the M15 stars exhibit a consis- tently lower fraction of stars with emission, than found in NGC 2808, a cluster which is more metal rich. This suggests the M15 chromospheres may have less material than stars at the same luminosity in NGC 2808 as demonstrated by detailed modeling of emission wings and consistent with the decrease in radius with metallicity.

Stars in M15 have $\mathrm{H} \alpha$ emission wings that vary in time so that the magnitude of the faintest giant showing emission changes among the different dates of observation. The lower limit to the presence of $\mathrm{H} \alpha$ emission in M15 $\left[\log \left(L / L_{\odot}\right)=2.36\right]$ is comparable to that found in NGC 2808 (Cacciari et al. 2004).

M15 exhibits continuous outflows at lower luminosities and with higher velocities than the more metal rich clusters, NGC 2808 and M4, hinting at a metallicity dependence. These outflows may decrease the chromospheric material and account for the lower fraction of stars with emission wings in M15. Detailed modeling is necessary to evaluate the mass loss rate from the line profiles. To identify mass loss as the solution to the second parameter problem will also require more complete measurements of clusters with varying metallicities.

The bisector velocity of the $\mathrm{H} \alpha$ core along the RGB indicates outflow (negative velocities) or no motion for stars brighter than $\log \left(L / L_{\odot}\right)=2.5$; the outflow velocity increases with increasing stellar luminosity. However, AGB stars near $\log \left(L / L_{\odot}\right) \sim 2.4$ have bisector velocities comparable in value to those at the tip of the RGB and also exhibit larger changes in velocity between observation than the RGB stars. We take this as evidence of more substantial and episodic mass outflow on the AGB.

Ca II K emission is detected at least to the limits of $\mathrm{H} \alpha$ emission; however, the asymmetry in the Ca II K core, where measurable, may differ from the asymmetry measured in the $\mathrm{H}$-alpha wings perhaps due to time variability or different line-forming regions.

Twelve stars identified in Spitzer observations as dusty IR sources and AGB stars (Bover et al. 2006) have radial velocities consistent with cluster membership. The similarities in $\mathrm{H} \alpha$ line profile characteristics between the Spitzer sources and other red giants in M15 suggests the IR emission attributed to circumstellar dust must be produced by an episodic process.

Observations reported here were obtained at the MMT Observatory, a joint facility of the Smithsonian Institution and the University of Arizona. We are grateful to the scientists at CfA who are developing and characterizing Hectochelle: Nelson Caldwell, Daniel G. Fabricant, Gabor Furesz, and David W. Latham. The authors also would like to thank John Roll and Maureen A. Conroy for developing SPICE software, Michael Calkins and Perry Berlind for their help during the observations, Robert Kurucz for the spectrum synthesis, and Martha Boyer for helpful comments on the manuscript. Kyle Cudworth kindly provided coordinates and photometry for M15 stars. Szabolcs Meszaros is supported in part by a SAO Predoctoral Fellowship, NASA, and the Hungarian OTKA Grant TS049872. This research is also supported in part by the Smithsonian Astrophysical Observatory. 


\section{REFERENCES}

Alonso, A., Arribas, S., \& Martínez-Roger, C. 1999, A\&AS, 140, 261

Alonso, A., Arribas, S., \& Martínez-Roger, C. 2001, A\&A, 376, 1039

Auriere, M., \& Cordoni, J. P. 1981, A\&AS, 46, 347

Baldry, I. K., Taylor, M. M., Bedding, T. R., \& Booth, A. J. 1997, MNRAS, 289, 979

Bates, B., Catney, M. G., \& Keenan, F. P. 1990, MNRAS, 245, 238

Bates, B., Kemp, S. N., \& Montgomery, A. S. 1993, A\&AS, 97, 937

Boyer, M. L., Woodward, C. E., van Loon, J. T., Gordon, K. D., Evans, A., Gehrz, R. D., Helton, L. A., \& Polomski, E. F. 2006, AJ, 132, 1415

Brown, A. 1951, ApJ, 113, 344

Buonanno, R., Corsi, C. E., Fusi Pecci, F., Richer, H. B., \& Fahlman, G. G. 1993, AJ, 105, 184

Buonanno, R., Corsi, C. E., Pulone, L., Fusi Pecci, F., \& Bellazzini, M. 1998 A\&A, 333, 505

Cacciari, C. et al. 2004, A\&A, 413, 343

Cacciari, C., \& Freeman, K. C. 1983, ApJ, 268, 185

Cardelli, J. A., Clayton, G. C., \& Mathis, J. S. 1989, ApJ, 345, 245

Carney, B. W., Latham, D. W., Stefanik, R. P., Laird, J. B., \& Morse, J. A. 2003, AJ, 125, 293

Catelan, M. 2000, ApJ, 531, 826

Catelan, M., Bellazzini, M., Landsman, W. B., Ferraro, F. R., Fusi Pecci, F., \& Galleti, S. 2001, AJ, 122, 3171

Cho, D. H., \& Lee, S. G. 2007, AJ, 133, 2163

Christy, R. F. 1966, ApJ, 144, 108

Coelho, P., Barbuy, B., Meléndez, J., Schiavon, R. P., \& Castilho, B. V. 2005, A\&A, 443, 735

Cohen, J. G. 1976, ApJ, 203, L127

Cudworth, K. M. 1976, AJ, 81, 519

Drukier, G. A., Slavin, S. D., Cohn, H. N., Lugger, P. M., Berrington, R. C., Murphy, B. W., \& Seitzer, P. O. 1998, AJ, 115,708

Dupree, A. K., Hartmann, L., \& Avrett, E. H. 1984, ApJ, 281, L37

Dupree, A. K., Hartmann, L., Smith, G. H., Rodgers, A. W. Roberts, W. H., \& Zucker, D. B. 1994, ApJ, 421, 542

Durrell, P. R., \& Harris, W. E. 1993, AJ, 105, 1420

Evans, A., Stickel, M., van Loon, J. T., Eyres, S. P. S., Hopwood, M. E. L., \& Penny, A. J. 2003, A\&A, 408, L9

Faulkner, D. J., Scott, T. R., Wood, P. R., \& Wright, A. E. 1991, ApJ, 374, 45

Freire, P. C., Kramer, M., Lyne, A. G., Camilo, F., Manchester, R. N., \& D'Amico, N. 2001, ApJ, 557, L105

Fusi Pecci, F., Ferraro, F. R., Bellazzini, M., Djorgovski, S., Piotto, G., \& Buonanno, R. 2001, AJ, 105, 1145

Gebhardt, K., Pryor, C., Williams, T. B., Hesser, J. E., \& Stetson, P. B. 1997, AJ, 113, 1026

Gratton, R. G., Pilachowski, C. A., \& Sneden, C. 1984, A\&A, 132,11

Harris, W. E. 1996, AJ, 112, 1487

Kemp, S. N., Bates, B. 1995, A\&AS, 112, 513

Kurucz, R. L. 1993, in ASPC 44: IAU Colloq. 138: Peculiar versus Normal Phenomena in A-type Related Stars, ed. Dworetsky, M. M., Castelli, F., \& Faraggiana, R. (San Francisco: ASP), 87

Kustner, F. 1921, Veroeffentlichungen des Astronomisches Institute der Universitaet Bonn, 15, 1

Lee, J. W., \& Carney, B. W. 1999, AJ, 118, 1373

Lee, Y. W., Demarque, P., \& Zinn, R. 1994, ApJ, 423, 248

Lyons, M. A., Kemp, S. N., Bates, B., \& Shaw, C. R. 1996, MNRAS, 280, 835

Mallia, E. A., \& Pagel, B. E. J. 1981, MNRAS, 194, 421

Mauas, P. J. D., Cacciari, C., \& Pasquini, L. 2006, A\&A, 454, 609

Mauas, P. J. D. 2007, in ASPC 368, Semiempirical Models of Solar and Stellar Active Chromospheres, ed. Heinzel, P. Dorotovič, I., \& Rutten, R. J. (San Francisco: ASP), 203 Mayor, M. et al. 1984, A\&A, 134, 118

Nardetto, N., Mourard, D., Kervella, P., Mathias, P., Mérand, A., \& Bersier, D. 2006, A\&A, 453, 309

Olofsson, H., González Delgado, D., Kerschbaum, F., \& Schöier, F. L. 2002, A\&A, 391, 1053
Origlia, L., Ferraro, F. R., Fusi Pecci, F., \& Rood, R. T. 2002 ApJ, 571, 458

Origlia, L., Rood, R. T., Fabbri, S., Ferraro, F. R., Fusi Pecci, F., \& Rich, R. M. 2007, ApJ, 667, L85

Peterson, R. C. 1981, ApJ, 248, L31

Peterson, R. C. 1981, ApJ, 258, 499

Peterson, R. C., Rood, R. T., \& Crocker, D. A. 1995, ApJ, 453, 214

Peterson, R. C., Seitzer, P., \& Cudworth, K. M. 1989, ApJ, 347, 251

Petterson, O. K. L., Cottrell, P. L., Albrow, M. D., \& Fokin, A. 2005, MNRAS, 362, 1167

Recio-Blanco, A., Aparicio, A., Piotto, G, de Angeli, F., \& Djorgovski, S. G. 2006, A\&A, 452, 875

Renzini, A. 1981, in IAU Colloq. 59, Effects of Mass Loss on Stellar Evolution, ed. C. Chiosi \& R. Stalio (Dordrecht:

Reidel), 319

Renzini, A., \& Fusi Pecci, F. 1988, ARAA, 26, 199

Sandage, A., \& Wallerstein, G. 1960, ApJ, 131, 598

Sandage, A., \& Wildey, R. 1967, ApJ, 150, 469

Sarajedini, A. 1997, AJ, 113, 682

Sarajedini, A., Chaboyer, B., \& Demarque, P. 1997, PASP, 109, 1321

Searle, L., \& Zinn, R. 1978, ApJ, 225, 357

Sills, A., \& Pinsonneault, M. H. 2000, ApJ, 540, 489

Skrutskie, M.F. et al. 2006, AJ, 131, 1163

Smith, G. H., \& Dupree, A. K. 1988, AJ, 95, 1547

Smith, G. H., Woodsworth, A. W., \& Hesser, J. E. 1995, MNRAS, 273, 632

Sneden, C., Kraft, R. P., Shetrone, M. D., Smith, G. H., Langer G. E., \& Prosser, C. F. 1997, AJ, 114, 1964

Sneden, C., Pilachowski, C. A., \& Kraft, R. P. 2000, AJ, 120, 1351

Soderberg, A. M., Pilachowski, C. A., Barden, S. C., Willmarth, D., \& Sneden, C. 1999, PASP, 111, 1233

Soker, N., Rappaport, S., \& Fregeau, J. 2001, ApJ, 563, L87

Stetson, P. B., Vandenberg, D. A., \& Bolte, M. 1996, PASP, 108, 560

Struck, C., Smith, D. C., Willson, L. A., Turner, G., \& Bowen, G. H. 2004, MNRAS, 353, 559

Sweigart, A. V. 1997, ApJ, 474, L23

Sweigart, A. V., Greggio, L., \& Renzini, A. 1990, ApJ, 364, 527

Szentgyorgyi, A. H., Cheimets, P., Eng, R, Fabricant, D. G., Geary, J. C., Hartmann, L., Pieri, M. R., \& Roll, J. B. 1998, in Proc. SPIE Vol. 3355, Optical Astronomical Instrumentation, ed. Sandro D'Odorico, 242

Taylor, J. H., \& Cordes, J. M. 1993, ApJ, 411, 674

Vandenberg, D. A., Bolte, M., \& Stetson, P. B. 1990, AJ, 100, 445 van Loon, J. T., Stanimirović, S., Evans, A., \& Muller, E. 2006, MNRAS, 365, 1277 


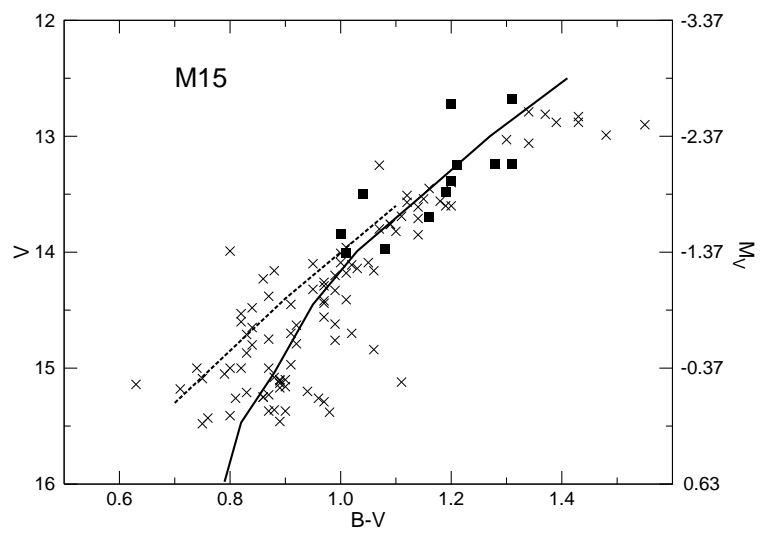

FIG. 1.- Color-magnitude diagram for all stars observed in 2005 and 2006. The solid line shows the fiducial curve of the RGB and the dashed line shows the fiducial curve of the AGB for M15 taken from observations of Durrell \& Harris (1993). Dusty giants identified with Spitzer Space Telescope (Boyer et al. 2006) and observed with Hectochelle, are denoted by squares. The redward "hook" seen among the brightest stars in M15 is not intrinsic to the stars but rather results from saturation of the photographic images (Sneden et al. 2000). Absolute magnitudes are obtained by assuming $(m-M)_{V}=15.37$ (Harris 1996).

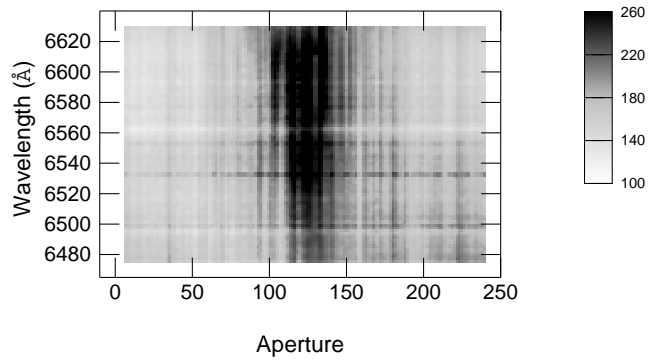

FIG. 2.- Variations in the intensity of the sky apertures in the $\mathrm{H} \alpha$ filter. Dark area indicates the highest count level. See scale at right. An anomalous intensity pattern occurs, which is currently not understood. Special extraction patterns were used for the sky fibers (see text).
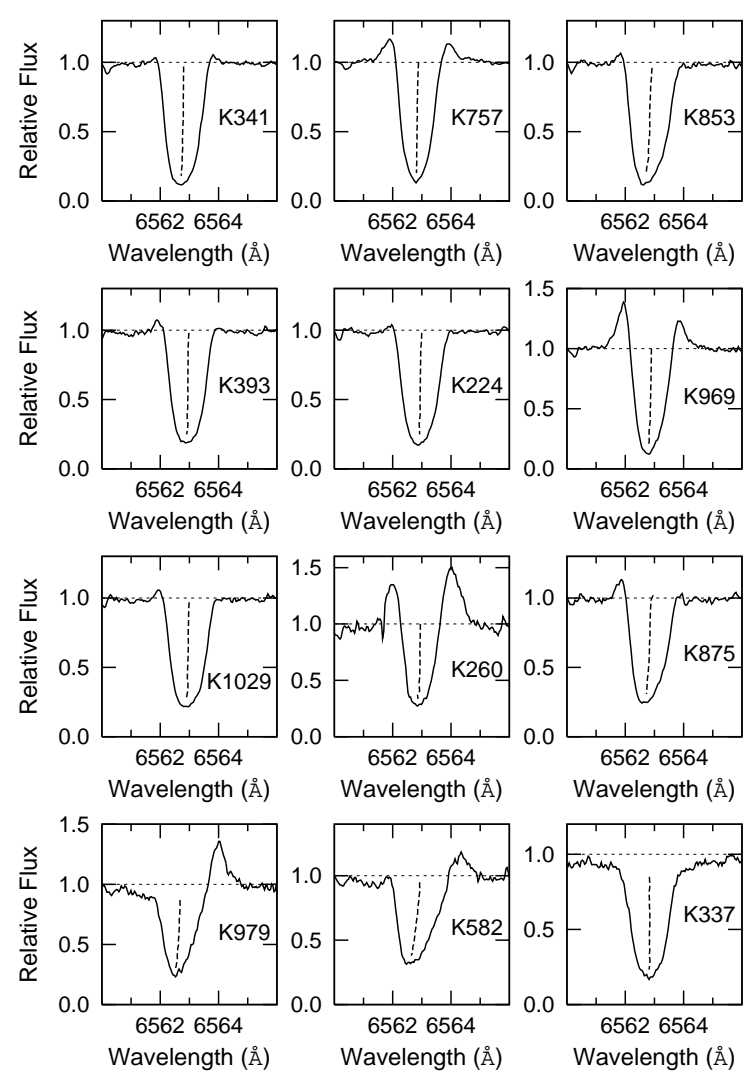

FiG. 3.- Normalized spectra of red giants in M15 which showed emission in $\mathrm{H} \alpha$ on 2005 May 22. The dashed line marks the bisector. The spectra are arranged in order of decreasing $\mathrm{V}$ magnitude; the brightest is at the top left and the stars become fainter from left to right. The wavelength scale is corrected for heliocentric velocity. K337 is an example of an $\mathrm{H} \alpha$ profile without emission. Stars K260 (Figures 3 and 4), K341 (Figures 3-6), K757 (Figures 3 and 4), and K969 (Figures 3 and 6) showed large variations in $\mathrm{H} \alpha$ emission during the observation period. 

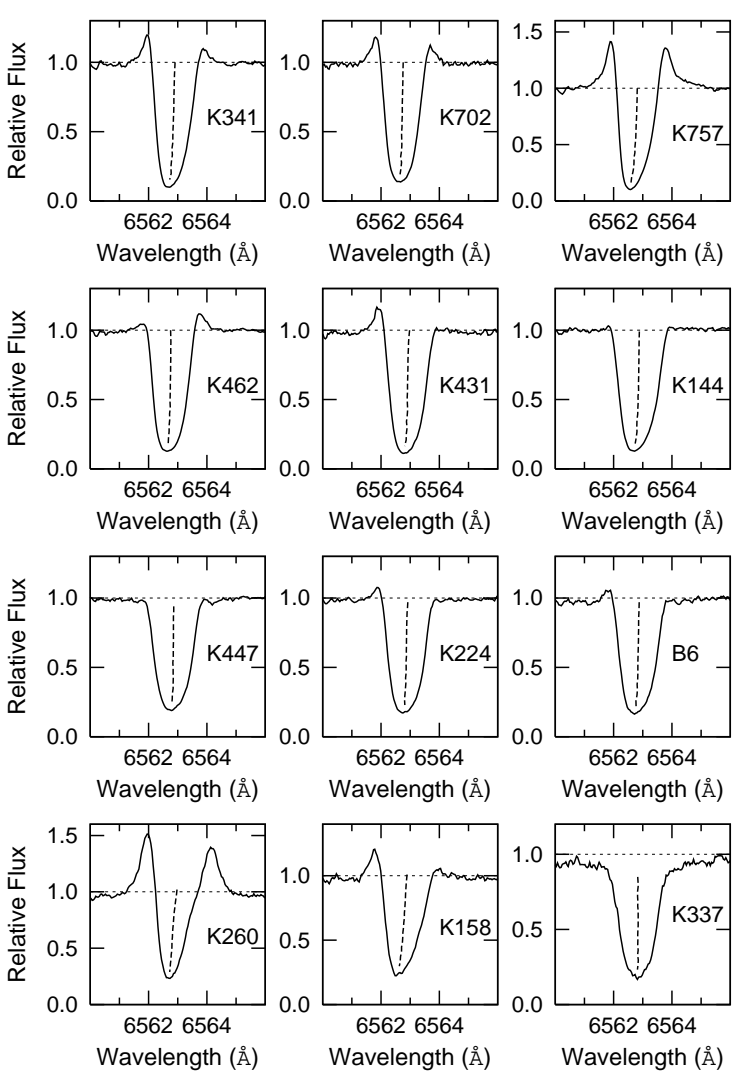

FIG. 4.- Normalized spectra of red giants in M15 which showed emission in $\mathrm{H} \alpha$ on 2006 May 11. For explanation please see Figure 3. Stars K260 (Figures 3 and 4), K341 (Figures 3-6), K431 (Figures 4 and 5), and K757 (Figures 3 and 4) showed large variations in $\mathrm{H} \alpha$ emission.
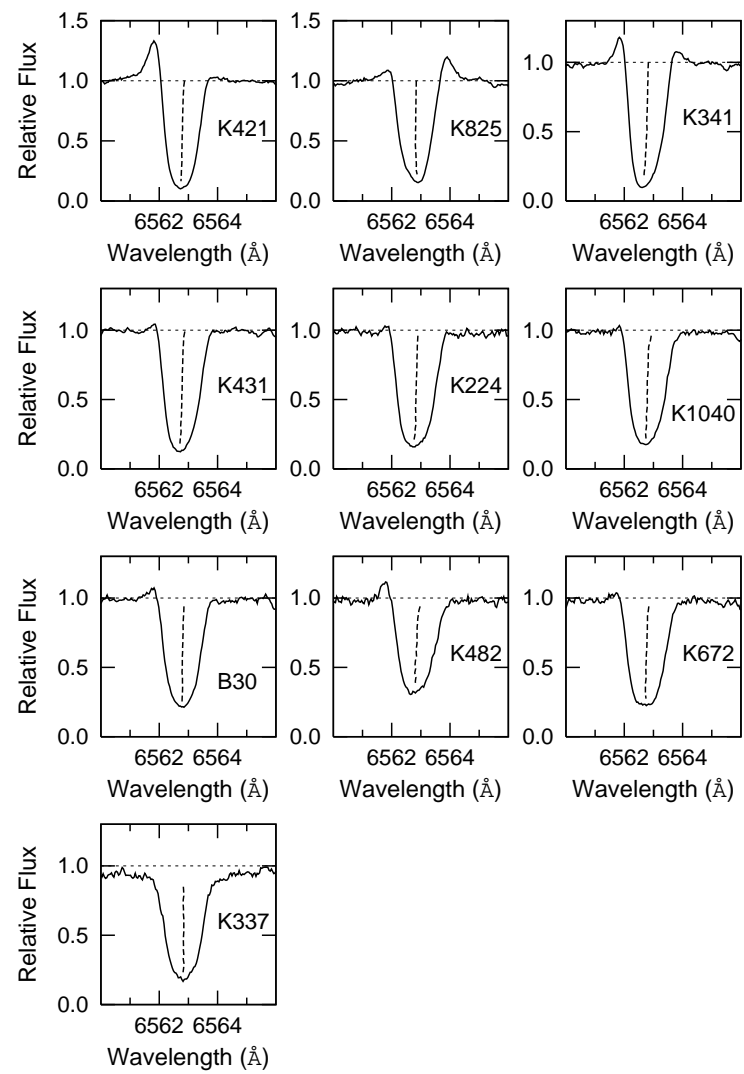

FiG. 5.- Normalized spectra of red giants in M15 which showed emission in $\mathrm{H} \alpha$ on 2006 October 4. For explanation please see Figure 3. Stars K341 (Figures 3-6), K431 (Figures 4 and 5) showed large variations in $\mathrm{H} \alpha$ emission. 

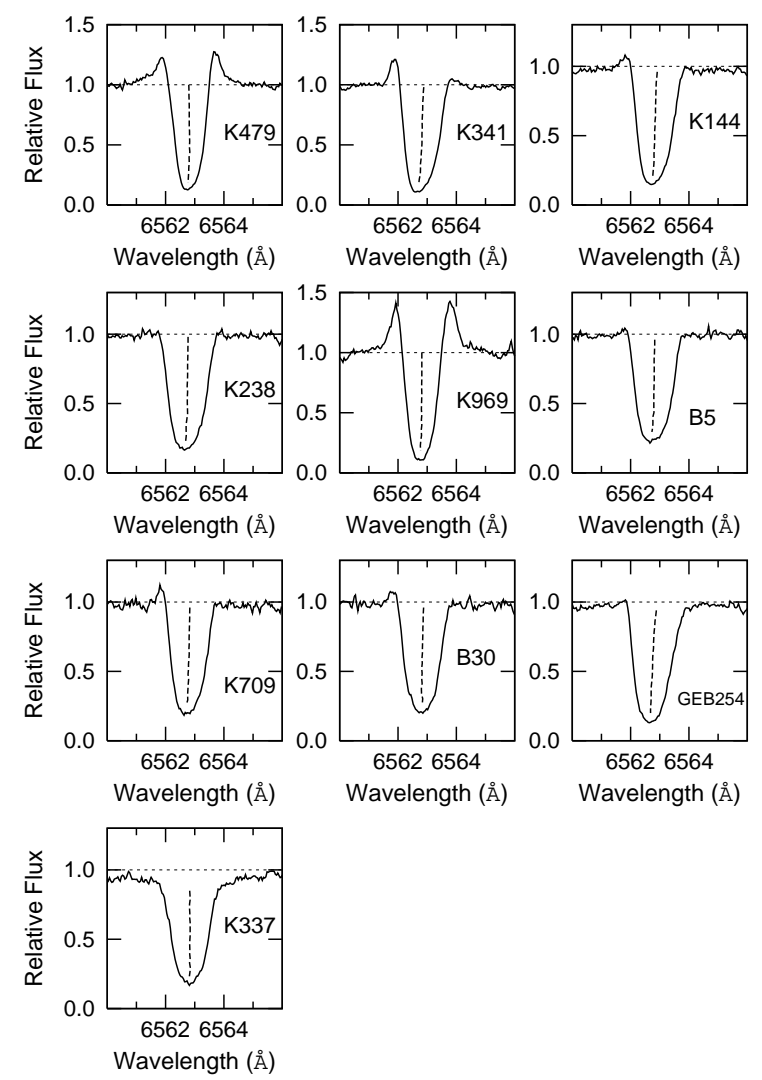

FIG. 6.- Normalized spectra of red giants in M15 which showed emission in $\mathrm{H} \alpha$ on 2006 October 7. For explanation please see Figure 3. Stars K341 (Figures 3-6), K969 (Figures 3 and 6) showed large variations in $\mathrm{H} \alpha$ emission.
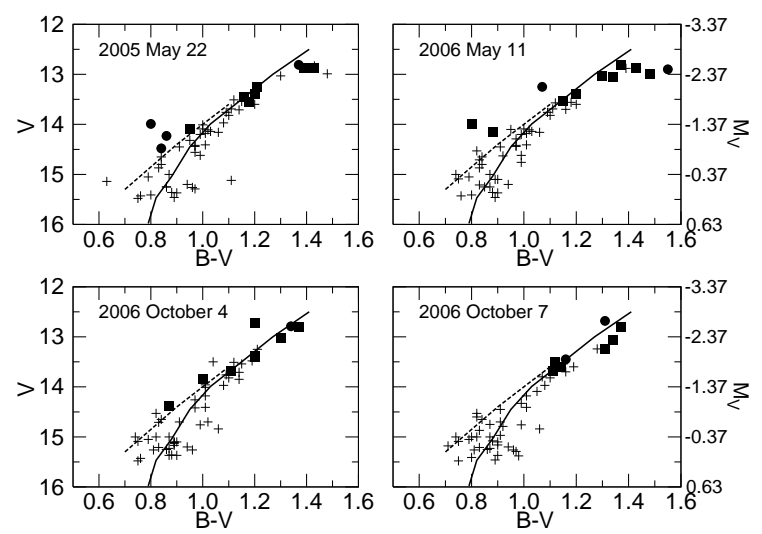

FIG. 7.- Color-magnitude diagrams for all M15 stars observed in 2005 and 2006. Stars with $H \alpha$ emission and with $\mathrm{B}<\mathrm{R}$ (indicating outflow) are marked with circles; stars with $\mathrm{B}>\mathrm{R}$ emission wings (suggests inflow) are denoted by squares. The solid line shows the fiducial curve of the RGB; dashed lines show the fiducial curve of the AGB for M15 from observations of Durrell \& Harris (1993).

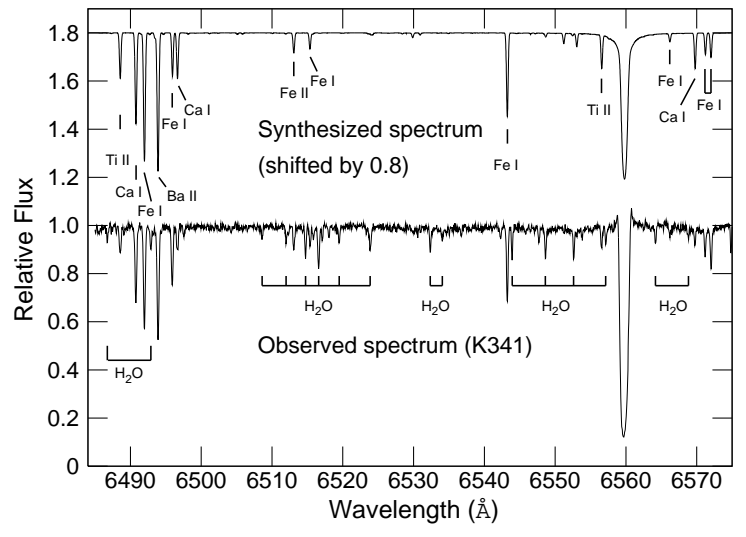

FIG. 8. - Kurucz synthesized spectrum shifted by 0.8 in relative flux and corrected for the star's radial velocity shown above the observed spectrum of K341. Atmospheric water vapor and other elements are marked.
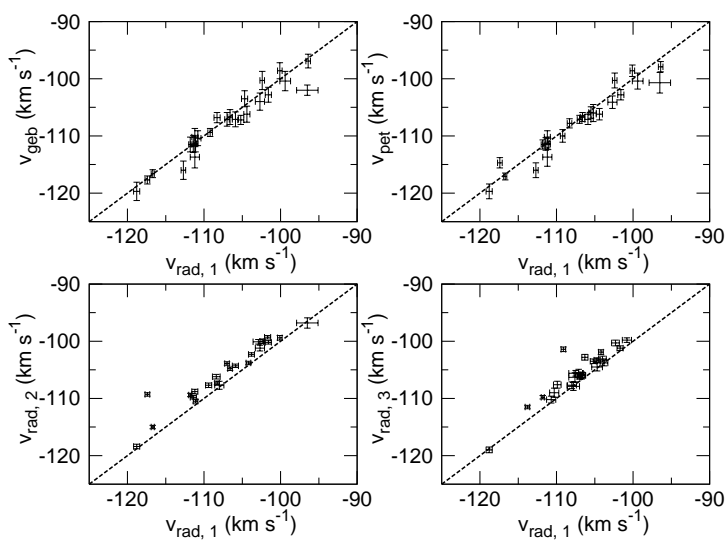

FIG. 9.- Top left: Radial velocities measured in this paper on 2005 May $22\left(v_{\text {rad,1 }}\right)$ for the same stars observed by Gebhardt et al. (1997) $\left(v_{g e b}\right)$. Top right: Radial velocities measured in this paper on 2005 May $22\left(v_{\text {rad, } 1}\right)$ for the same stars observed by Peterson et al. (1989) $\left(v_{p e t}\right)$. There is good agreement between observations taken on 2005 May 22 and observations for the same stars from Gebhardt et al. (1997) and Peterson et al (1989). Lower left: Radial velocity measured with Hectochelle for the same stars observed on 2005 May $22\left(v_{\text {rad,1 }}\right)$ compared to 2006 May $11\left(v_{\text {rad }, 2}\right)$. The velocity offset between 2006 May 11 and 2005 May 22 is $+1.9 \pm 0.5 \mathrm{~km} \mathrm{~s}^{-1}$. Lower right: Radial velocities for the same stars measured with Hectochelle on 2005 May $22\left(v_{\text {rad,1 }}\right)$ compared to 2006 October $4\left(v_{\text {rad }, 3}\right)$. The velocity offset between 2006 October 4 and 2005 May 22 is $+0.9 \pm 0.5 \mathrm{~km} \mathrm{~s}^{-1}$. The dashed line marks a 1:1 relation. The offsets are applied to our radial velocities for the 2006 May and 2006 October spectra (see text). 

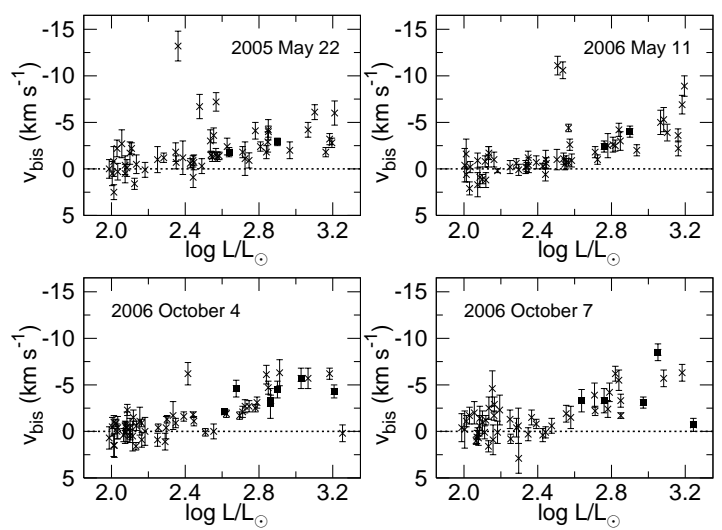

FIG. 10. - The velocity difference $\left(v_{b i s}\right)$ between the top and the bottom of the bisector of $\mathrm{H} \alpha$ as a function of luminosity. Negative values indicate a blueshifted core (outward motion), positive values denote a red shifted core (inward motion). A predominant outward motion sets in near $\log L / L \odot \approx 2.4$ and increases in velocity towards more luminous stars. Dusty giants identified with Spitzer Space Telescope (Boyer et al. 2006) and observed with Hectochelle, are denoted by squares. See text for discussion of the outlying stars between $\log L / L_{\odot}=2.3$ and 2.6 with velocities more negative than $-10 \mathrm{~km} \mathrm{~s}^{-1}$ which appear to be AGB stars.

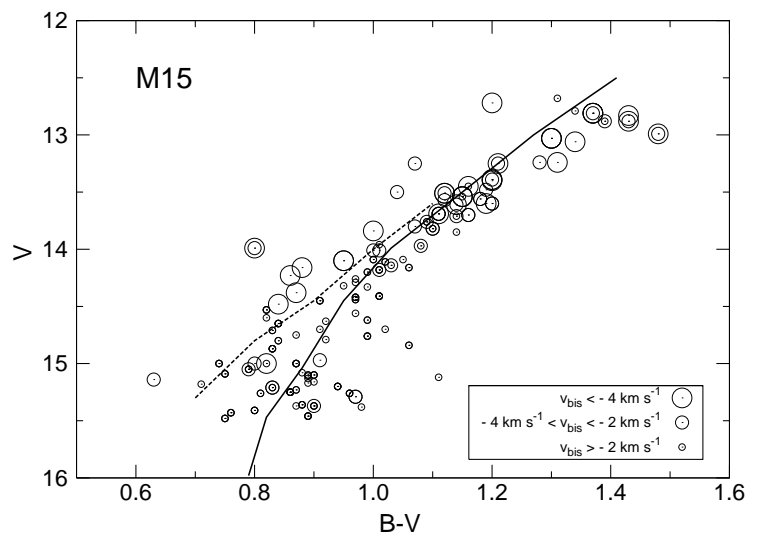

FIG. 11.- Color-magnitude diagram for all M15 stars observed in 2005 and 2006, where the size of the circle indicates the velocity of the $\mathrm{H} \alpha$ bisector asymmetry. Big circle: $v_{b i s}<-4 \mathrm{~km} \mathrm{~s}^{-1}$, medium circle: $-4 \mathrm{~km} \mathrm{~s}^{-1}<v_{\text {bis }}<-2 \mathrm{~km} \mathrm{~s}^{-1}$, small circle: $v_{b i s}>-2 \mathrm{~km} \mathrm{~s}^{-1}$. Concentric circles generally indicate multiple observations of the same star.
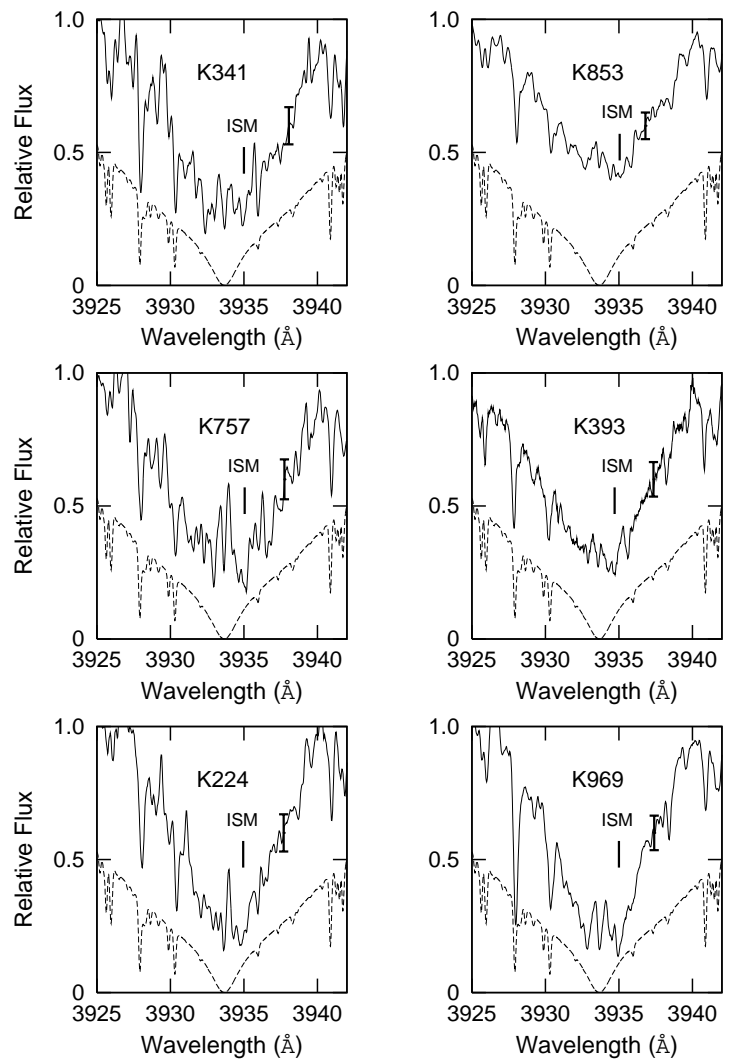

FIG. 12.- Spectra of some red giants in M15 which showed emission in Ca II K. The observed spectra are shifted up by 0.1 . The Kurucz model of K341 is denoted by a dashed line. The spectra are arranged in order of decreasing V magnitude; the brightest is at the top left and the stars become fainter from left to right. The spectra are smoothed to make the spectral features more visible. Error bars show the photon noise in the original, unsmoothed spectra. The line marked ISM denotes absorption by the interstellar medium. 

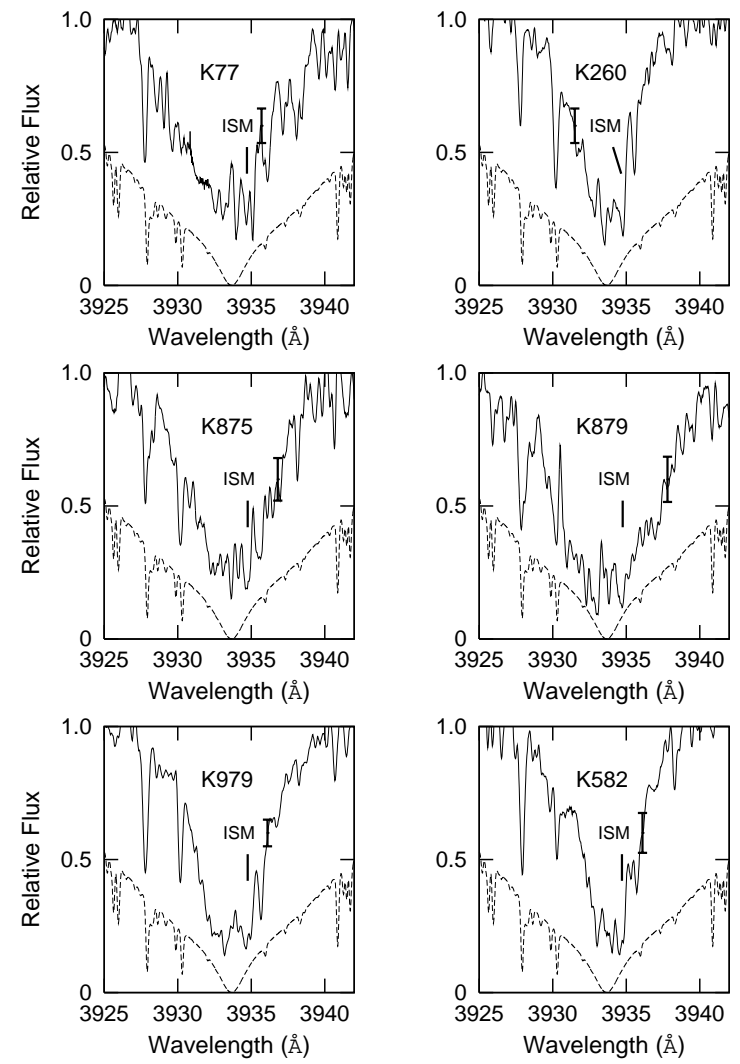

FIG. 13. - Spectra of some red giants in M15 which showed emission in Ca II K. For explanation please see Figure 12.

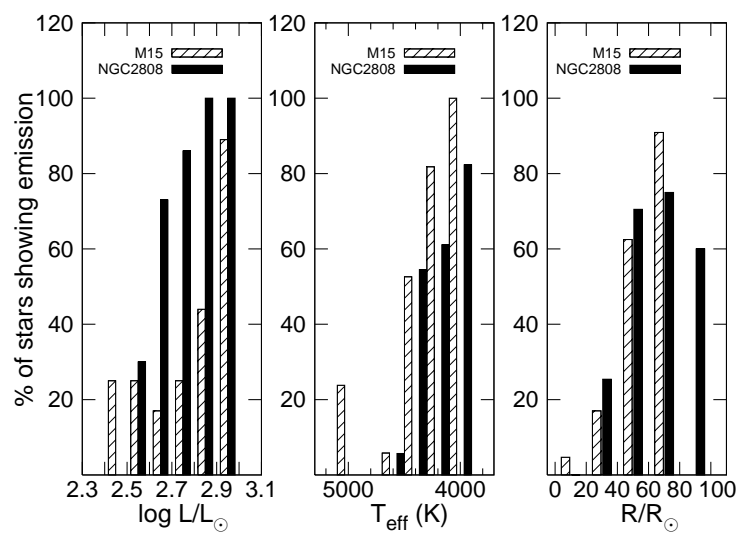

FIG. 14.- Left panel: Percentage of stars showing emission in M15 and NGC 2808 as a function of luminosity. Values for the $\log \left(L / L_{\odot}\right)=2.9$ bin include all brighter stars as well. Center panel: Percentage of stars showing emission in M15 and NGC 2808 as a function of effective temperature. The lowest and highest bin includes the lower and upper limits respectively. Right panel: Percentage of stars showing emission in M15 and NGC 2808 as a function of stellar radius. 
TABLE 1

Photometric Data of Observed Cluster Members

\begin{tabular}{|c|c|c|c|c|c|c|c|c|c|c|}
\hline ID No. ${ }^{a}$ & $R A(2000)^{\mathrm{b}}$ & $\operatorname{Dec}(2000)^{b}$ & $\mathrm{~B}$ & $\mathrm{~V}$ & $\mathrm{~J}$ & $\mathrm{H}$ & $\mathrm{K}$ & $B-V$ & $\mathrm{~V}-\mathrm{K}$ & Obs. ${ }^{\mathrm{c}}$ \\
\hline B5 ${ }^{d}$ & 212908.43 & +120911.8 & 14.63 & 13.51 & 11.368 & 10.726 & 10.645 & 1.12 & 2.87 & $1,3,4$ \\
\hline$B 6^{d}$ & 212912.36 & +121049.8 & 14.69 & 13.54 & 11.411 & 10.775 & 10.656 & 1.15 & 2.88 & $1,2,3,4$ \\
\hline $\mathrm{B} 16^{\mathrm{d}}$ & 212953.12 & +121231.1 & 14.85 & 13.76 & 11.732 & 11.154 & 11.029 & 1.09 & 2.73 & 3 \\
\hline B30 d & 213044.13 & +121122.6 & 14.80 & 13.69 & 11.496 & 10.927 & 10.807 & 1.11 & 2.88 & $1,2,3,4$ \\
\hline $\mathrm{C} 3{ }^{\mathrm{e}}$ & 212913.06 & +121115.0 & 15.49 & 14.65 & 12.698 & 12.156 & 12.039 & 0.84 & 2.61 & $1,2,3,4$ \\
\hline $\mathrm{C} 20^{\mathrm{e}}$ & 212952.32 & +121939.7 & 16.35 & 15.46 & 13.646 & 13.089 & 12.991 & 0.89 & 2.47 & $1,2,3,4$ \\
\hline $\mathrm{C} 35^{\mathrm{e}}$ & 213049.28 & +120731.2 & 15.77 & 15.14 & 13.398 & 12.965 & 12.855 & 0.63 & 2.29 & 1 \\
\hline GEB $254^{\mathrm{f}}$ & 212958.15 & +120946.7 & $14.55^{\mathrm{g}}$ & $13.24^{\mathrm{g}}$ & 9.560 & 9.964 & 9.777 & 1.31 & 3.46 & 4,5 \\
\hline GEB $289^{\mathrm{f}}$ & 212959.37 & +121002.9 & $14.54^{\mathrm{g}}$ & $13.50^{\mathrm{g}}$ & 11.079 & 10.449 & 10.311 & 1.04 & 3.12 & 3,5 \\
\hline K12 & 212930.77 & +120632.7 & 15.84 & 15.05 & 13.386 & 12.886 & 12.851 & 0.79 & 2.20 & $1,2,3,4$ \\
\hline K21 & 212933.12 & +121251.0 & 15.74 & 15.00 & 13.323 & 12.834 & 12.726 & 0.74 & 2.27 & $2,3,4$ \\
\hline K22 & 212933.53 & +120455.3 & 15.42 & 14.41 & 12.482 & 11.938 & 11.809 & 1.01 & 2.60 & $1,2,3,4$ \\
\hline K26 & 212934.62 & +120319.2 & 15.87 & 15.00 & 13.197 & 12.693 & 12.566 & 0.87 & 2.43 & $1,2,3,4$ \\
\hline K27 & 212935.08 & +120603.7 & 15.84 & 15.09 & 13.451 & 12.994 & 12.894 & 0.75 & 2.20 & $2,3,4$ \\
\hline K31 & 212935.87 & +120827.3 & 16.04 & 15.21 & 13.529 & 13.006 & 12.882 & 0.83 & 2.33 & $2,3,4$ \\
\hline K42 & 212938.00 & +121158.2 & 16.10 & 15.23 & 13.452 & 12.925 & 12.887 & 0.87 & 2.34 & 3,4 \\
\hline K47 & 212938.72 & +121153.0 & 15.13 & 14.11 & 12.152 & 11.588 & 11.488 & 1.02 & 2.62 & 1,2 \\
\hline K56 & 212940.04 & +121600.2 & 16.27 & 15.37 & 13.651 & 13.107 & 12.966 & 0.90 & 2.40 & $1,2,3,4$ \\
\hline K60 & 212941.25 & +120719.4 & 16.07 & 15.26 & 13.532 & 12.991 & 12.928 & 0.81 & 2.33 & 3,4 \\
\hline K64 & 212942.96 & +120953.4 & 16.11 & 15.25 & 13.504 & 13.003 & 12.884 & 0.86 & 2.37 & $1,2,3,4$ \\
\hline K69 & 212943.77 & +120833.3 & 15.36 & 14.45 & 12.501 & 11.969 & 11.854 & 0.91 & 2.60 & $1,2,4$ \\
\hline K70 & 212943.59 & +121547.4 & 15.27 & 14.32 & 12.338 & 11.746 & 11.653 & 0.95 & 2.67 & 1 \\
\hline K77 & 212944.65 & +120730.8 & 14.92 & 13.82 & 11.752 & 11.148 & 11.026 & 1.10 & 2.79 & $1,2,3,4$ \\
\hline K87 & 212945.81 & +120845.5 & 14.87 & 13.80 & 11.776 & 11.225 & 11.075 & 1.07 & 2.73 & 4 \\
\hline K89 & 212946.07 & +121131.5 & 15.35 & 14.53 & 12.564 & 12.055 & 11.955 & 0.82 & 2.58 & $2,3,4$ \\
\hline K92 & 212946.70 & +120320.7 & 16.14 & 15.20 & 13.359 & 12.848 & 12.791 & 0.94 & 2.41 & $1,2,3,4$ \\
\hline K105 & 212947.39 & +120904.7 & 16.26 & 15.29 & 13.477 & 13.023 & 12.940 & 0.97 & 2.35 & 1,4 \\
\hline K112 & 212947.78 & +121130.7 & 15.96 & 15.08 & 13.167 & 12.654 & 12.592 & 0.88 & 2.49 & 4 \\
\hline K114 & 212947.87 & +120845.4 & 14.99 & 13.85 & 11.781 & 11.245 & 11.080 & 1.14 & 2.77 & 3 \\
\hline K129 & 212948.63 & +121145.8 & 15.23 & 14.26 & 12.292 & 11.725 & 11.620 & 0.97 & 2.64 & 3 \\
\hline K133 & 212948.84 & +121025.3 & 15.99 & 15.10 & 13.435 & 13.014 & 12.898 & 0.89 & 2.20 & 2,3 \\
\hline K136 & 212949.13 & +120903.7 & 15.80 & 15.00 & 13.217 & 12.811 & 12.707 & 0.80 & 2.29 & 4 \\
\hline K137 & 212949.45 & +120827.0 & 16.36 & 15.38 & 13.628 & 13.134 & 13.059 & 0.98 & 2.32 & 3 \\
\hline K144 & 212949.79 & +121105.9 & 14.40 & 13.06 & 10.745 & 10.053 & 9.944 & 1.34 & 3.12 & 2,4 \\
\hline K145 & 212949.80 & +121229.9 & 16.24 & 15.36 & 13.557 & 13.090 & 12.957 & 0.88 & 2.40 & $1,2,3$ \\
\hline K146 & 212949.94 & +120805.3 & 14.69 & 13.57 & 11.427 & 10.843 & 10.726 & 1.12 & 2.84 & 2 \\
\hline K151 & 212950.14 & +120752.2 & 16.00 & 15.11 & 13.303 & 12.757 & 12.699 & 0.89 & 2.61 & 3 \\
\hline K152 & 212950.16 & +120640.7 & 16.11 & 15.25 & 13.553 & 12.999 & 12.877 & 0.86 & 2.37 & $1,2,3$ \\
\hline K153 & 212949.92 & +121812.1 & 16.23 & 15.48 & 13.684 & 13.195 & 13.067 & 0.75 & 2.41 & $1,3,4$ \\
\hline K158 & 212950.29 & +120902.8 & 15.04 & 14.16 & 12.380 & 11.879 & 11.806 & 0.88 & 2.35 & 2 \\
\hline K202 & 212951.89 & +120638.9 & 16.06 & 15.17 & 13.420 & 12.906 & 12.838 & 0.89 & 2.33 & 3 \\
\hline K224 & 212952.31 & +121051.5 & 14.59 & 13.39 & 11.235 & 10.672 & 10.515 & 1.20 & 2.88 & $1,2,3,5$ \\
\hline K238 & 212952.65 & +121044.0 & 14.52 & 13.24 & 11.041 & 10.429 & 10.305 & 1.28 & 2.94 & 4,5 \\
\hline $\mathrm{K} 255$ & 212953.12 & +121231.1 & 14.85 & 13.76 & 11.732 & 11.154 & 11.029 & 1.09 & 2.73 & 1,2 \\
\hline K260 & 212953.31 & +120934.0 & 14.79 & 13.99 & 12.486 & 12.125 & 12.053 & 0.80 & 1.94 & 1,2 \\
\hline K272 & 212953.57 & +120910.7 & 14.67 & 13.48 & 11.307 & 10.736 & 10.606 & 1.19 & 2.87 & 3,5 \\
\hline K288 & 212953.79 & +121020.3 & 14.85 & 13.71 & 11.667 & 11.120 & 10.966 & 1.14 & 2.74 & 1,3 \\
\hline K328 & 212954.73 & +120859.2 & 14.86 & 13.70 & 11.591 & 11.065 & 10.906 & 1.16 & 2.80 & $2,4,5$ \\
\hline K337 & 212955.05 & +120248.5 & 15.75 & 14.76 & 12.863 & 12.311 & 12.200 & 0.99 & 2.56 & $2,3,4$ \\
\hline K341 & 212954.93 & +121322.5 & 14.18 & 12.81 & 10.455 & 9.796 & 9.695 & 1.37 & 3.12 & $1,2,3,4$ \\
\hline K361 & 212955.29 & $\begin{array}{r}+120913.7\end{array}$ & 14.97 & 13.96 & 12.029 & 11.475 & 11.364 & 1.01 & 2.60 & 3 \\
\hline K393 & 212955.73 & +121133.8 & 14.46 & 13.25 & 11.042 & 10.452 & 10.315 & 1.21 & 2.94 & 1,2 \\
\hline K421 & 212956.18 & +121017.9 & 13.92 & 12.72 & 10.414 & 9.781 & 9.649 & 1.20 & 3.07 & 3,5 \\
\hline K431 & 212956.18 & +121233.8 & 14.33 & 13.03 & 10.759 & 10.144 & 10.039 & 1.30 & 2.99 & $1,2,3$ \\
\hline K447 & 212956.45 & +121029.4 & 14.32 & 13.25 & 11.132 & 10.613 & 10.468 & 1.07 & 2.78 & 2 \\
\hline K462 & 212956.67 & +120946.3 & 14.45 & 12.90 & 10.534 & 9.860 & 9.722 & 1.55 & 3.18 & 2 \\
\hline K476 & 212956.72 & +121310.5 & 15.55 & 14.63 & 12.714 & 12.202 & 12.130 & 0.92 & 2.50 & 2 \\
\hline K479 & 212956.79 & +121027.0 & 13.99 & 12.68 & 10.276 & 9.678 & 9.524 & 1.31 & 3.16 & 4,5 \\
\hline K482 & 212956.94 & +120844.7 & 15.25 & 14.38 & 12.632 & 12.195 & 12.071 & 0.87 & 2.31 & 3 \\
\hline K506 & 212957.43 & +120821.5 & 15.62 & 14.75 & 12.848 & 12.299 & 12.232 & 0.87 & 2.52 & 4 \\
\hline K550 & 212958.03 & +121154.2 & 16.00 & 15.10 & 13.357 & 12.833 & 12.742 & 0.90 & 2.36 & $2,3,4$ \\
\hline K567 & 212958.33 & +120912.8 & 14.46 & 13.25 & 11.129 & 10.508 & 10.414 & 1.21 & 2.84 & 3,5 \\
\hline K582 & 212958.60 & +120808.0 & 15.32 & 14.48 & 12.829 & 12.370 & 12.305 & 0.84 & 2.18 & 1 \\
\hline K583 & 212958.57 & +120921.4 & 14.26 & 12.83 & 10.315 & 9.726 & 9.569 & 1.43 & 3.26 & 1 \\
\hline K647 & 212959.46 & $\begin{array}{r}+120835.6 \\
\end{array}$ & 14.79 & 13.60 & 11.388 & 10.807 & 10.686 & 1.19 & 2.91 & 4 \\
\hline K654 & 212959.53 & +121152.6 & 16.06 & 15.16 & 13.350 & 12.861 & 12.785 & 0.90 & 2.38 & 4 \\
\hline K672 & 212959.81 & +121110.7 & 14.84 & 13.84 & 11.837 & 11.290 & 11.211 & 1.00 & 2.63 & 3,5 \\
\hline K677 & 212959.99 & +120626.6 & 15.82 & 15.00 & 13.330 & 12.824 & 12.811 & 0.82 & 2.19 & 3,4 \\
\hline K691 & 213000.03 & +121339.5 & 15.64 & 14.80 & 12.859 & 12.321 & 12.217 & 0.84 & 2.58 & 1,2 \\
\hline K702 & 213000.34 & +121050.9 & 14.47 & 12.99 & 10.609 & 10.080 & 9.910 & 1.48 & 3.08 & 1,2 \\
\hline K709 & 213000.38 & $\begin{array}{r}+120736.4 \\
\end{array}$ & 14.75 & 13.61 & 11.535 & 10.944 & 10.847 & 1.14 & 2.76 & 3,4 \\
\hline K736 & 213000.63 & +120928.4 & 15.02 & 14.01 & 12.063 & 11.474 & 11.346 & 1.01 & 2.66 & 3,5 \\
\hline K757 & 213000.91 & +120857.1 & 14.31 & 12.88 & 10.383 & 9.759 & 9.605 & 1.43 & 3.28 & 1,2 \\
\hline K800 & 213001.65 & +121230.3 & 16.23 & 15.12 & 13.029 & 12.575 & 12.761 & 1.11 & 2.36 & 1 \\
\hline K825 & 213002.25 & +121121.5 & 14.13 & 12.79 & 10.227 & 9.582 & 9.433 & 1.34 & 3.36 & 3 \\
\hline
\end{tabular}


TABLE 1

Photometric Data of Observed Cluster Members

\begin{tabular}{|c|c|c|c|c|c|c|c|c|c|c|}
\hline K846 & 213002.78 & +120655.7 & 15.05 & 13.97 & 11.918 & 11.388 & 11.247 & 1.08 & 2.72 & $1,4,5$ \\
\hline K853 & 213002.74 & +121043.9 & 14.27 & 12.88 & 10.469 & 9.860 & 9.727 & 1.39 & 3.15 & 1,2 \\
\hline K866 & 213003.09 & +121021.8 & 15.72 & 14.70 & 12.885 & 12.322 & 12.269 & 1.02 & 2.43 & 3 \\
\hline K875 & 213003.17 & +121328.7 & 15.05 & 14.10 & 12.180 & 11.659 & 11.506 & 0.95 & 2.60 & 1,2 \\
\hline K879 & 213003.50 & +120312.5 & 15.22 & 14.16 & 12.205 & 11.615 & 11.527 & 1.06 & 2.63 & 1,2 \\
\hline K902 & 213004.00 & +120857.8 & 15.90 & 14.84 & 13.003 & 12.483 & 12.364 & 1.06 & 2.48 & 3,4 \\
\hline K906 & 213004.09 & +120727.1 & 16.24 & 15.37 & 13.560 & 13.046 & 13.009 & 0.87 & 2.36 & 3 \\
\hline K919 & 213004.32 & +121056.2 & 14.80 & 13.60 & 11.459 & 10.893 & 10.757 & 1.20 & 2.84 & 1,2 \\
\hline K925 & 213004.62 & +120853.7 & 15.61 & 14.62 & 12.733 & 12.231 & 12.067 & 0.99 & 2.55 & 1,2 \\
\hline K926 & 213004.65 & +120740.5 & 15.89 & 15.18 & 13.591 & 13.186 & 13.107 & 0.71 & 2.07 & 4 \\
\hline K932 & 213004.75 & +121110.3 & 15.14 & 14.09 & 12.099 & 11.534 & 11.438 & 1.05 & 2.65 & 4 \\
\hline K947 & 213005.18 & +121320.3 & 15.26 & 14.29 & 12.311 & 11.724 & 11.613 & 0.97 & 2.68 & 2 \\
\hline K954 & 213005.54 & +120855.3 & 15.32 & 14.33 & 12.357 & 11.835 & 11.719 & 0.99 & 2.61 & 4 \\
\hline K969 & 213006.37 & +120659.3 & 14.61 & 13.45 & 11.364 & 10.829 & 10.700 & 1.16 & 2.75 & 1,4 \\
\hline K979 & 213006.96 & +120746.5 & 15.09 & 14.23 & 12.454 & 11.978 & 11.898 & 0.86 & 2.33 & 1 \\
\hline K989 & 213007.30 & +121050.7 & 16.02 & 15.13 & 13.556 & 13.078 & 12.950 & 0.89 & 2.18 & 3 \\
\hline K993 & 213007.40 & +121033.1 & 15.01 & 14.01 & 11.964 & 11.399 & 11.264 & 1.00 & 2.75 & 1 \\
\hline K1010 & 213008.42 & +120942.1 & 15.88 & 14.97 & 13.167 & 12.630 & 12.545 & 0.91 & 2.43 & 4 \\
\hline K1014 & 213008.95 & +120849.1 & 15.61 & 14.70 & 13.003 & 12.483 & 12.364 & 0.91 & 2.34 & 3 \\
\hline K1029 & 213009.71 & +121342.4 & 14.74 & 13.56 & 11.349 & 10.739 & 10.601 & 1.18 & 2.96 & 1,2 \\
\hline K1030 & 213009.78 & +121254.4 & 15.17 & 14.14 & 12.109 & 11.538 & 11.401 & 1.03 & 2.74 & 1,2 \\
\hline K1033 & 213009.89 & +121052.5 & 15.41 & 14.44 & 12.447 & 11.882 & 11.773 & 0.97 & 2.67 & 1,2 \\
\hline K1040 & 213010.49 & +121006.2 & 14.60 & 13.40 & 11.151 & 10.562 & 10.438 & 1.20 & 2.96 & 3 \\
\hline K1049 & 213011.31 & +120148.5 & 15.53 & 14.56 & 12.604 & 12.038 & 11.934 & 0.97 & 2.63 & 1 \\
\hline K1054 & 213011.38 & +120841.2 & 15.19 & 14.20 & 12.181 & 11.626 & 11.517 & 0.99 & 2.68 & 1,2 \\
\hline K1056 & 213011.69 & +121033.7 & 15.71 & 14.79 & 12.907 & 12.384 & 12.227 & 0.92 & 2.56 & 4 \\
\hline K1069 & 213014.26 & +120923.4 & 15.42 & 14.60 & 12.660 & 12.125 & 12.006 & 0.82 & 2.60 & 4 \\
\hline K1073 & 213014.95 & +121020.7 & 15.19 & 14.18 & 12.127 & 11.593 & 11.465 & 1.01 & 2.72 & $1,2,3,4$ \\
\hline K1074 & 213015.23 & +121134.5 & 16.22 & 15.26 & 13.406 & 12.910 & 12.786 & 0.96 & 2.47 & $1,3,4$ \\
\hline K1079 & 213015.66 & +120822.9 & 15.09 & 14.09 & 12.061 & 11.517 & 11.408 & 1.00 & 2.68 & 1,2 \\
\hline K1083 & 213015.78 & +121659.6 & 16.19 & 15.43 & 13.566 & 13.053 & 12.987 & 0.76 & 2.44 & $1,2,3$ \\
\hline K1084 & 213016.06 & +121334.3 & 15.39 & 14.42 & 12.415 & 11.860 & 11.772 & 0.97 & 2.65 & $1,2,3$ \\
\hline K1097 & 213021.04 & +121300.8 & 16.21 & 15.41 & 13.631 & 13.127 & 13.025 & 0.80 & 2.39 & $1,2,3,4$ \\
\hline K1106 & 213022.71 & +121759.6 & 15.54 & 14.71 & 12.719 & 12.163 & 12.044 & 0.83 & 2.67 & 2,3 \\
\hline K1136 & 213031.78 & +120854.8 & 15.70 & 14.87 & 13.005 & 12.483 & 12.329 & 0.83 & 2.54 & $1,2,3,4$ \\
\hline
\end{tabular}

Note. - The visual photometry is taken from Cudworth (1976); $\mathrm{J}, \mathrm{H}, \mathrm{K}$ photometry is taken from the 2MASS Catalog (Skrutskie et al. 2006).

${ }^{a}$ Kustner (1921) is the identification for the majority of the stars denoted by $\mathrm{K}$.

b 2MASS coordinates (Skrutskie et al. 2006).

c Observations: 1: 2005 May 22; 2: 2006 May 11; 3: 2006 October 4; 4: 2006 October 7; 5: Dusty giants identified by Boyer et al. (2006).

$\mathrm{d}$ Brown (1951).

e Cudworth (1976).

$\mathrm{f}$ Gebhardt et al. (1997).

$\mathrm{g}$ B and V magnitudes are taken from Auriere \& Cordoni (1981). 
TABLE 2

Hectochelle Observations of M15

\begin{tabular}{ccccc}
\hline \hline $\begin{array}{c}\text { Date } \\
(\mathrm{UT})\end{array}$ & $\begin{array}{c}\text { Total exp. } \\
(\mathrm{s})\end{array}$ & $\begin{array}{c}\text { Wavelength } \\
(\AA)\end{array}$ & Filter Name & $\begin{array}{c}\text { Number of } \\
\text { Observed Stars }\end{array}$ \\
\hline 2005 May 22 (Field 1) & $3 \times 1200$ & $6485-6575$ & OB25 & 53 \\
2005 May 23 (Field 1) & $3 \times 1200$ & $3910-3990$ & Ca41 & 53 \\
2006 May 11 (Field 2) & $3 \times 2100$ & $6475-6630$ & OB25 & 54 \\
2006 October 4 (Field 3) & $3 \times 2100$ & $6475-6630$ & OB25 & 58 \\
2006 October 7 (Field 4) & $3 \times 2100$ & $6475-6630$ & OB25 & 50 \\
\hline
\end{tabular}


TABLE 3

Physical Parameters of Cluster Members

\begin{tabular}{|c|c|c|c|c|c|c|c|}
\hline ID No. & $M_{V}$ & $(B-V)_{0}$ & $(V-K)_{0}$ & $\mathrm{P}^{\mathrm{a}}$ & $\begin{array}{c}T_{\text {eff }} \\
(\mathrm{K})\end{array}$ & $\log L / L_{\odot}$ & $R / R_{\odot}$ \\
\hline B5 & -1.86 & 1.02 & 2.595 & 99 & 4490 & 2.850 & 42.9 \\
\hline B6 & -1.83 & 1.05 & 2.605 & 98 & 4480 & 2.840 & 42.6 \\
\hline B16 & -1.61 & 0.99 & 2.455 & 0 & 4610 & 2.724 & 35.2 \\
\hline B30 & -1.68 & 1.01 & 2.605 & 99 & 4480 & 2.780 & 39.7 \\
\hline C3 & -0.72 & 0.74 & 2.335 & 97 & 4730 & 2.348 & 21.7 \\
\hline $\mathrm{C} 20$ & +0.09 & 0.79 & 2.195 & 97 & 4870 & 2.004 & 13.8 \\
\hline C35 & -0.23 & 0.53 & 2.015 & 0 & 5090 & 2.109 & 14.2 \\
\hline GEB 254 & -2.13 & 1.21 & 3.195 & $\ldots$ & 4080 & 3.052 & 65.5 \\
\hline GEB 289 & -1.87 & 0.94 & 2.845 & $\ldots$ & 4300 & 2.864 & 47.5 \\
\hline K12 & -0.32 & 0.69 & 1.925 & 99 & 5200 & 2.135 & 14.0 \\
\hline $\mathrm{K} 21$ & -0.37 & 0.64 & 1.995 & 72 & 5110 & 2.163 & 15.0 \\
\hline $\mathrm{K} 22$ & -0.96 & 0.91 & 2.325 & 96 & 4740 & 2.443 & 24.1 \\
\hline K26 & -0.37 & 0.77 & 2.155 & 92 & 4920 & 2.182 & 16.5 \\
\hline $\mathrm{K} 27$ & -0.28 & 0.65 & 1.925 & 88 & 5200 & 2.119 & 13.8 \\
\hline K31 & -0.16 & 0.73 & 2.055 & 76 & 5040 & 2.086 & 14.1 \\
\hline K42 & -0.14 & 0.77 & 2.065 & 85 & 5020 & 2.079 & 14.1 \\
\hline $\mathrm{K} 47$ & -1.26 & 0.92 & 2.345 & 99 & 4720 & 2.566 & 28.0 \\
\hline K56 & +0.00 & 0.80 & 2.125 & 93 & 4950 & 2.030 & 13.7 \\
\hline K60 & -0.11 & 0.71 & 2.055 & 86 & 5040 & 2.066 & 13.8 \\
\hline K64 & -0.12 & 0.76 & 2.095 & 75 & 4990 & 2.074 & 14.2 \\
\hline K69 & -0.92 & 0.81 & 2.325 & 99 & 4740 & 2.427 & 23.6 \\
\hline K70 & -1.05 & 0.85 & 2.395 & 3 & 4670 & 2.490 & 26.2 \\
\hline K77 & -1.55 & 1.00 & 2.515 & 99 & 4560 & 2.711 & 35.4 \\
\hline K87 & -1.57 & 0.97 & 2.455 & 99 & 4610 & 2.708 & 34.5 \\
\hline K89 & -0.84 & 0.72 & 2.305 & 99 & 4760 & 2.392 & 22.5 \\
\hline K92 & -0.17 & 0.84 & 2.135 & 95 & 4940 & 2.100 & 14.9 \\
\hline K105 & -0.08 & 0.87 & 2.075 & 89 & 5010 & 2.056 & 13.8 \\
\hline K112 & -0.29 & 0.78 & 2.215 & 94 & 4850 & 2.158 & 16.6 \\
\hline K114 & -1.52 & 1.04 & 2.495 & 99 & 4580 & 2.695 & 34.5 \\
\hline K129 & -1.11 & 0.87 & 2.365 & 99 & 4700 & 2.509 & 26.4 \\
\hline K133 & -0.27 & 0.79 & 1.925 & 82 & 5200 & 2.115 & 13.7 \\
\hline K136 & -0.37 & 0.70 & 2.015 & 53 & 5090 & 2.165 & 15.2 \\
\hline K137 & +0.01 & 0.88 & 2.045 & 93 & 5050 & 2.016 & 13.0 \\
\hline K144 & -2.31 & 1.24 & 2.845 & 99 & 4300 & 3.083 & 61.1 \\
\hline K145 & -0.01 & 0.78 & 2.125 & 83 & 4950 & 2.034 & 13.8 \\
\hline K146 & -1.80 & 1.02 & 2.565 & 99 & 4520 & 2.820 & 40.9 \\
\hline K151 & -0.26 & 0.79 & 2.335 & 92 & 4730 & 2.164 & 17.5 \\
\hline K152 & -0.12 & 0.76 & 2.095 & 76 & 4990 & 2.074 & 14.2 \\
\hline K153 & +0.11 & 0.65 & 2.135 & 62 & 4940 & 1.988 & 13.1 \\
\hline K158 & -1.21 & 0.78 & 2.075 & 99 & 5010 & 2.508 & 23.2 \\
\hline K202 & -0.20 & 0.79 & 2.055 & 64 & 5040 & 2.102 & 14.4 \\
\hline $\mathrm{K} 224$ & -1.98 & 1.10 & 2.605 & 99 & 4480 & 2.900 & 45.6 \\
\hline K238 & -2.13 & 1.18 & 2.665 & 99 & 4390 & 2.972 & 51.6 \\
\hline $\mathrm{K} 255$ & -1.61 & 0.99 & 2.455 & 99 & 4610 & 2.724 & 35.2 \\
\hline K260 & -1.38 & 0.70 & 1.665 & 99 & 5590 & 2.536 & 19.3 \\
\hline $\mathrm{K} 272$ & -1.89 & 1.09 & 2.595 & 99 & 4460 & 2.862 & 44.1 \\
\hline K288 & -1.66 & 1.04 & 2.465 & 99 & 4600 & 2.746 & 36.2 \\
\hline K328 & -1.67 & 1.06 & 2.525 & 99 & 4550 & 2.760 & 37.6 \\
\hline K337 & -0.61 & 0.89 & 2.285 & 92 & 4780 & 2.297 & 20.0 \\
\hline K341 & -2.56 & 1.27 & 2.845 & 99 & 4300 & 3.183 & 68.6 \\
\hline K361 & -1.41 & 0.91 & 2.325 & 99 & 4740 & 2.623 & 29.6 \\
\hline K393 & -2.12 & 1.11 & 2.665 & 99 & 4430 & 2.967 & 50.4 \\
\hline K421 & -2.65 & 1.10 & 2.795 & 99 & 4330 & 3.207 & 69.5 \\
\hline $\mathrm{K} 431$ & -2.34 & 1.20 & 2.715 & 99 & 4390 & 3.066 & 57.5 \\
\hline $\mathrm{K} 447$ & -2.12 & 0.97 & 2.505 & 99 & 4570 & 2.937 & 45.7 \\
\hline K462 & -2.47 & 1.45 & 2.905 & 99 & 4260 & 3.161 & 68.1 \\
\hline $\mathrm{K} 476$ & -0.74 & 0.82 & 2.225 & 98 & 4840 & 2.340 & 20.5 \\
\hline K479 & -2.69 & 1.21 & 2.885 & 99 & 4270 & 3.244 & 74.6 \\
\hline K482 & -0.99 & 0.77 & 2.035 & 99 & 5060 & 2.415 & 20.5 \\
\hline K506 & -0.62 & 0.77 & 2.245 & 99 & 4820 & 2.295 & 19.6 \\
\hline K550 & -0.27 & 0.80 & 2.085 & 93 & 5000 & 2.133 & 15.1 \\
\hline K567 & -2.32 & 1.11 & 2.565 & 99 & 4520 & 3.028 & 51.9 \\
\hline K582 & -0.89 & 0.74 & 1.905 & 99 & 5230 & 2.361 & 18.0 \\
\hline K583 & -2.54 & 1.33 & 2.985 & 99 & 4200 & 3.210 & 74.2 \\
\hline K647 & -1.77 & 1.09 & 2.635 & 99 & 4460 & 2.821 & 42.0 \\
\hline K654 & -0.21 & 0.80 & 2.105 & 89 & 4980 & 2.112 & 14.9 \\
\hline K672 & -1.53 & 0.90 & 2.355 & 99 & 4710 & 2.676 & 31.9 \\
\hline K677 & -0.37 & 0.72 & 1.915 & 98 & 5220 & 2.154 & 14.2 \\
\hline K691 & -0.57 & 0.74 & 2.305 & 99 & 4760 & 2.284 & 19.9 \\
\hline K702 & -2.38 & 1.38 & 2.805 & 99 & 4330 & 3.102 & 61.6 \\
\hline K709 & -1.76 & 1.04 & 2.485 & 99 & 4590 & 2.789 & 38.2 \\
\hline K736 & -1.36 & 0.91 & 2.385 & 99 & 4680 & 2.613 & 30.0 \\
\hline K757 & -2.49 & 1.33 & 3.005 & 99 & 4190 & 3.195 & 73.2 \\
\hline K800 & -0.25 & 1.01 & 2.085 & 65 & 5000 & 2.125 & 15.0 \\
\hline K825 & -2.58 & 1.24 & 3.085 & 99 & 4140 & 3.253 & 80.2 \\
\hline
\end{tabular}


TABLE 3

Physical Parameters of Cluster Members

\begin{tabular}{llllllll} 
K846 & -1.40 & 0.98 & 2.445 & 99 & 4620 & 2.638 & 31.7 \\
K853 & -2.49 & 1.29 & 2.875 & 99 & 4280 & 3.162 & 67.6 \\
K866 & -0.67 & 0.92 & 2.155 & 86 & 4920 & 2.302 & 19.0 \\
K875 & -1.27 & 0.85 & 2.325 & 99 & 4740 & 2.567 & 27.8 \\
K879 & -1.21 & 0.96 & 2.355 & 98 & 4710 & 2.547 & 27.5 \\
K902 & -0.53 & 0.96 & 2.205 & 97 & 4860 & 2.253 & 18.4 \\
K906 & +0.00 & 0.77 & 2.085 & 88 & 5000 & 2.025 & 13.4 \\
K919 & -1.77 & 1.10 & 2.565 & 99 & 4520 & 2.808 & 40.3 \\
K925 & -0.75 & 0.89 & 2.275 & 96 & 4790 & 2.351 & 21.2 \\
K926 & -0.19 & 0.61 & 1.795 & 84 & 5390 & 2.070 & 12.1 \\
K932 & -1.28 & 0.95 & 2.375 & 99 & 4690 & 2.579 & 28.8 \\
K947 & -1.08 & 0.87 & 2.405 & 99 & 4660 & 2.504 & 26.7 \\
K954 & -1.04 & 0.89 & 2.335 & 99 & 4730 & 2.476 & 25.1 \\
K969 & -1.92 & 1.06 & 2.475 & 99 & 4590 & 2.851 & 41.1 \\
K979 & -1.14 & 0.76 & 2.055 & 99 & 5040 & 2.478 & 22.2 \\
K989 & -0.24 & 0.79 & 1.905 & 73 & 5230 & 2.101 & 13.3 \\
K993 & -1.36 & 0.90 & 2.475 & 99 & 4590 & 2.627 & 31.7 \\
K1010 & -0.40 & 0.81 & 2.155 & 97 & 4920 & 2.194 & 16.8 \\
K1014 & -0.67 & 0.81 & 2.065 & 98 & 5020 & 2.291 & 18.0 \\
K1029 & -1.81 & 1.08 & 2.685 & 99 & 4420 & 2.848 & 44.1 \\
K1030 & -1.23 & 0.93 & 2.465 & 99 & 4600 & 2.574 & 29.7 \\
K1033 & -0.93 & 0.87 & 2.395 & 99 & 4670 & 2.442 & 24.8 \\
K1040 & -1.97 & 1.10 & 2.685 & 99 & 4420 & 2.912 & 47.5 \\
K1049 & -0.81 & 0.87 & 2.355 & 0 & 4710 & 2.387 & 22.9 \\
K1054 & -1.17 & 0.89 & 2.405 & 99 & 4660 & 2.540 & 27.9 \\
K1056 & -0.58 & 0.82 & 2.285 & 94 & 4780 & 2.285 & 19.7 \\
K1069 & -0.77 & 0.72 & 2.325 & 99 & 4740 & 2.367 & 22.1 \\
K1073 & -1.19 & 0.91 & 2.445 & 99 & 4620 & 2.554 & 28.8 \\
K1074 & -0.11 & 0.86 & 2.195 & 88 & 4870 & 2.084 & 15.1 \\
K1079 & -1.28 & 0.90 & 2.405 & 99 & 4660 & 2.584 & 29.3 \\
K1083 & +0.06 & 0.66 & 2.165 & 66 & 4910 & 2.012 & 13.7 \\
K1084 & -0.95 & 0.87 & 2.375 & 99 & 4690 & 2.447 & 24.7 \\
K1097 & +0.04 & 0.70 & 2.115 & 50 & 4970 & 2.013 & 13.3 \\
K1106 & -0.66 & 0.73 & 2.395 & 96 & 4670 & 2.334 & 21.9 \\
K1136 & -0.50 & 0.73 & 2.265 & 79 & 4800 & 2.250 & 18.8 \\
& & & & & & & \\
\hline
\end{tabular}

a Membership probability from proper motion observations (Cudworth 1976). 
TABLE 4

B/R ratio of H $\alpha$ Line for Stars with Emission Wings

\begin{tabular}{|c|c|c|c|c|}
\hline ID No. & $\bar{B} B / R 2005$ May 22 & $\bar{B} / R 2006$ May 11 & $B / R 2006$ October 4 & 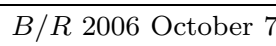 \\
\hline B5 & no emission & .. & no emission & $>1$ \\
\hline B6 & no emission & $>1$ & no emission & no emission \\
\hline B30 & no emission & no emission & $>1$ & $>1$ \\
\hline GEB 254 & $\ldots$ & $\ldots$ & $\ldots$ & $>1$ \\
\hline K144 & $\ldots$ & $>1$ & no emission & $>1$ \\
\hline K158 & $\ldots$ & $>1$ & $\ldots$ & $\ldots$ \\
\hline K224 & $>1$ & $>1$ & $>1$ & $\ldots$ \\
\hline $\mathrm{K} 238$ & $\ldots$ & $\ldots$ & $\ldots$ & $>1$ \\
\hline K260 & $<1$ & $>1$ & $\ldots$ & $\ldots$ \\
\hline K341 & $<1$ & $>1$ & $>1$ & $>1$ \\
\hline K393 & $>1$ & $\ldots$ & $\ldots$ & $\ldots$ \\
\hline K421 & $\ldots$ & $\ldots$ & $>1$ & $\ldots$ \\
\hline K431 & no emission & $>1$ & $>1$ & $\ldots$ \\
\hline K447 & $\ldots$ & $<1$ & $\ldots$ & $\ldots$ \\
\hline K462 & $\ldots$ & $<1$ & $\ldots$ & $\ldots$ \\
\hline K479 & $\ldots$ & $\ldots$ & $\ldots$ & $<1$ \\
\hline K482 & $\ldots$ & $\ldots$ & $>1$ & $\ldots$ \\
\hline K582 & $<1$ & $\ldots$ & $\ldots$ & $\ldots$ \\
\hline K672 & $\ldots$ & $\ldots$ & $>1$ & $\ldots$ \\
\hline K702 & no emission & $>1$ & $\ldots$ & $\ldots$ \\
\hline K709 & $\cdots$ & $\cdots$ & $\cdots$ & $>1$ \\
\hline K757 & $>1$ & $>1$ & $\ldots$ & $\ldots$ \\
\hline K825 & $\cdots$ & $\cdots$ & $<1$ & $\cdots$ \\
\hline K853 & $>1$ & no emission & $\ldots$ & $\cdots$ \\
\hline K875 & $>1$ & no emission & $\ldots$ & $\ldots$ \\
\hline K969 & $>1$ & $\ldots$ & $\ldots$ & $<1$ \\
\hline K979 & $<1$ & $\ldots$ & $\cdots$ & $\cdots$ \\
\hline K1029 & $>1$ & no emission & $\cdots$ & $\cdots$ \\
\hline K1040 & $\cdots$ & & $>1$ & $\cdots$ \\
\hline
\end{tabular}

Note. - The parameter $\mathrm{B} / \mathrm{R}$ is the intensity ratio of Blue (short wavelength) and Red (long wavelength) emission peaks. The symbol $\cdots$ indicates the star was not observed. If $B / R$ ratio is $>1$ the line profile indicates inflow, if $B / R$ ratio is $<1$ the line profile indicates outflow. 
TABLE 5

Radial Velocity of Cluster Members

\begin{tabular}{|c|c|c|c|c|}
\hline ID No. & $\begin{array}{l}v_{\text {rad }, 1}{ }^{\mathrm{a}} \\
\left(\mathrm{km} \mathrm{s}^{-1}\right)\end{array}$ & $\begin{array}{l}v_{\text {rad }, 2}{ }^{\mathrm{a}} \\
\left(\mathrm{km} \mathrm{s} \mathrm{s}^{-1}\right)\end{array}$ & $\begin{array}{l}v_{\text {rad }, 3}{ }^{\mathrm{a}} \\
\left(\mathrm{km} \mathrm{s} \mathrm{s}^{-1}\right)\end{array}$ & $\begin{array}{l}v_{\text {rad }, 4}{ }^{\mathrm{a}} \\
\left(\mathrm{km} \mathrm{s}^{-1}\right)\end{array}$ \\
\hline B5 & $-109.1 \pm 0.3$ & $\ldots$ & $-102.3 \pm 0.4$ & $-108.4 \pm 0.3$ \\
\hline B6 & $-113.8 \pm 0.3$ & $-113.3 \pm 0.3$ & $-112.4 \pm 0.3$ & $-113.3 \pm 0.4$ \\
\hline B16 & $\ldots$ & $\ldots$ & $-103.4 \pm 0.4$ & $\ldots$ \\
\hline B30 & $-104.2 \pm 0.3$ & $-105.7 \pm 0.3$ & $-102.8 \pm 0.4$ & $-104.2 \pm 0.3$ \\
\hline C3 & $-102.3 \pm 0.5$ & $-101.3 \pm 0.4$ & $-101.2 \pm 0.5$ & $-101.1 \pm 0.5$ \\
\hline $\mathrm{C} 20$ & $-108.0 \pm 0.6$ & $-109.6 \pm 0.7$ & $-108.6 \pm 0.6$ & $-108.6 \pm 0.7$ \\
\hline C35 & $-106.8 \pm 0.6$ & $\ldots$ & $\ldots$ & $\ldots$ \\
\hline GEB 254 & $\ldots$ & $\ldots$ & $\ldots$ & $-100.7 \pm 0.3$ \\
\hline GEB 289 & $\ldots$ & $\ldots$ & $-100.4 \pm 0.5$ & $\ldots$ \\
\hline $\mathrm{K} 12$ & $-107.6 \pm 0.8$ & $-107.0 \pm 0.6$ & $-107.2 \pm 0.8$ & $-107.0 \pm 0.9$ \\
\hline $\mathrm{K} 21$ & $\ldots$ & $-112.0 \pm 0.7$ & $-111.0 \pm 0.8$ & $-112.9 \pm 0.7$ \\
\hline $\mathrm{K} 22$ & $-101.7 \pm 0.4$ & $-101.2 \pm 0.3$ & $-102.1 \pm 0.4$ & $-102.0 \pm 0.4$ \\
\hline $\mathrm{K} 26$ & $-104.0 \pm 0.5$ & $-104.0 \pm 0.4$ & $-104.0 \pm 0.5$ & $-103.7 \pm 0.5$ \\
\hline $\mathrm{K} 27$ & $\ldots$ & $-107.4 \pm 0.6$ & $-106.9 \pm 0.7$ & $-107.2 \pm 0.8$ \\
\hline K31 & $\ldots$ & $-105.3 \pm 0.4$ & $-105.5 \pm 0.5$ & $-105.8 \pm 0.6$ \\
\hline $\mathrm{K} 42$ & $\cdots$ & $\ldots$ & $-104.5 \pm 0.7$ & $-104.3 \pm 0.8$ \\
\hline $\mathrm{K} 47$ & $-102.9 \pm 0.7$ & $-102.0 \pm 0.4$ & $\ldots$ & $\ldots$ \\
\hline K56 & $-104.7 \pm 0.7$ & $-104.4 \pm 0.5$ & $-105.4 \pm 0.7$ & $-104.3 \pm 0.7$ \\
\hline K60 & $\ldots$ & $\ldots$ & $-109.5 \pm 0.5$ & $-109.2 \pm 0.6$ \\
\hline K64 & $-107.8 \pm 0.8$ & $-108.6 \pm 0.6$ & $-108.8 \pm 0.7$ & $-108.9 \pm 0.7$ \\
\hline K69 & $-102.7 \pm 0.6$ & $-103.1 \pm 0.5$ & $\ldots$ & $-103.1 \pm 0.6$ \\
\hline K70 & $-109.2 \pm 0.4$ & $\ldots$ & $\ldots$ & $\ldots$ \\
\hline K77 & $-104.7 \pm 0.4$ & $-103.7 \pm 0.3$ & $-104.2 \pm 0.4$ & $-104.3 \pm 0.4$ \\
\hline K87 & $\ldots$ & $\ldots$ & $\ldots$ & $-108.3 \pm 0.4$ \\
\hline K89 & $\ldots$ & $-109.5 \pm 0.4$ & $-109.8 \pm 0.5$ & $-109.8 \pm 0.6$ \\
\hline K92 & $-107.5 \pm 0.9$ & $-106.7 \pm 0.3$ & $-106.5 \pm 0.5$ & $-106.1 \pm 0.6$ \\
\hline K105 & $-112.3 \pm 0.5$ & $\ldots$ & $\ldots$ & $-112.2 \pm 0.6$ \\
\hline $\mathrm{K} 112$ & $\ldots$ & $\ldots$ & $\ldots$ & $-100.3 \pm 0.5$ \\
\hline K114 & $\ldots$ & $\ldots$ & $-113.2 \pm 0.4$ & $\ldots$ \\
\hline K129 & $\ldots$ & $\ldots$ & $-102.5 \pm 0.4$ & $\ldots$ \\
\hline K133 & $\ldots$ & $-104.3 \pm 0.5$ & $-104.6 \pm 0.7$ & $\ldots$ \\
\hline K136 & $\ldots$ & $\ldots$ & $\ldots$ & $-111.0 \pm 0.7$ \\
\hline K137 & $\ldots$ & $\ldots$ & $-111.5 \pm 0.6$ & $\ldots$ \\
\hline K144 & $\ldots$ & $-108.6 \pm 0.3$ & $\ldots$ & $-110.9 \pm 0.3$ \\
\hline K145 & $-110.7 \pm 0.6$ & $-110.7 \pm 0.4$ & $-111.1 \pm 0.6$ & $\ldots$ \\
\hline K146 & $\ldots$ & $-101.3 \pm 0.3$ & $\ldots$ & $\ldots$ \\
\hline K151 & $\ldots$ & $\ldots$ & $-94.1 \pm 0.5$ & $\ldots$ \\
\hline K152 & $-100.8 \pm 0.6$ & $-100.0 \pm 0.4$ & $-100.7 \pm 0.4$ & $\ldots$ \\
\hline K153 & $-110.3 \pm 0.6$ & $\ldots$ & $-109.9 \pm 0.8$ & $-111.0 \pm 0.7$ \\
\hline $\mathrm{K} 158$ & $\ldots$ & $-110.5 \pm 0.5$ & $\cdots$ & $\cdots$ \\
\hline K202 & $\cdots$ & $\ldots$ & $-99.4 \pm 0.7$ & $\cdots$ \\
\hline $\mathrm{K} 224$ & $-106.6 \pm 0.3$ & $-106.7 \pm 0.3$ & $-106.8 \pm 0.4$ & $\ldots$ \\
\hline $\mathrm{K} 238$ & $\ldots$ & $\ldots$ & $\ldots$ & $-102.0 \pm 0.3$ \\
\hline $\mathrm{K} 255$ & $-102.4 \pm 0.3$ & $-101.9 \pm 0.4$ & $\ldots$ & $\ldots$ \\
\hline $\mathrm{K} 260$ & $-96.5 \pm 1.4$ & $-98.7 \pm 0.9$ & $\ldots$ & $\ldots$ \\
\hline $\mathrm{K} 272$ & $\ldots$ & $\ldots$ & $-106.2 \pm 0.4$ & $\cdots$ \\
\hline $\mathrm{K} 288$ & $-105.2 \pm 0.4$ & $\ldots$ & $-104.4 \pm 0.4$ & $\ldots$ \\
\hline K328 & $\ldots$ & $-102.9 \pm 0.3$ & $\ldots$ & $-102.4 \pm 0.4$ \\
\hline K337 & $\ldots$ & $-107.2 \pm 0.4$ & $-107.8 \pm 0.6$ & $-107.8 \pm 0.6$ \\
\hline K341 & $-111.8 \pm 0.2$ & $-111.3 \pm 0.3$ & $-110.9 \pm 0.3$ & $-110.9 \pm 0.3$ \\
\hline K361 & $\cdots$ & $\ldots$ & $-108.6 \pm 0.5$ & $\cdots$ \\
\hline K393 & $-96.4 \pm 0.3$ & $\cdots$ & $\ldots$ & $\ldots$ \\
\hline $\mathrm{K} 421$ & $\ldots$ & $\ldots$ & $-111.7 \pm 0.3$ & $\ldots$ \\
\hline $\mathrm{K} 431$ & $-107.0 \pm 0.3$ & $-105.8 \pm 0.3$ & $-107.1 \pm 0.4$ & $\ldots$ \\
\hline $\mathrm{K} 447$ & $\cdots$ & $-105.2 \pm 0.3$ & $\cdots$ & $\cdots$ \\
\hline $\mathrm{K} 462$ & $\cdots$ & $-113.4 \pm 0.3$ & $\cdots$ & $\cdots$ \\
\hline $\mathrm{K} 476$ & $\ldots$ & $-109.1 \pm 0.5$ & $\ldots$ & $\ldots$ \\
\hline K479 & $\ldots$ & $\ldots$ & $\cdots$ & $-122.5 \pm 0.4$ \\
\hline K482 & $\ldots$ & $\ldots$ & $-110.2 \pm 0.5$ & $\ldots$ \\
\hline K506 & $\ldots$ & $\ldots$ & $\ldots$ & $-103.5 \pm 0.5$ \\
\hline K550 & $\cdots$ & $-111.1 \pm 0.5$ & $-110.2 \pm 0.7$ & $-110.2 \pm 0.7$ \\
\hline K567 & $\ldots$ & $\ldots$ & $-93.42 \pm 0.4$ & $\ldots$ \\
\hline K582 & $-99.4 \pm 0.7$ & $\ldots$ & $\ldots$ & $\ldots$ \\
\hline K583 & $-109.2 \pm 0.3$ & $\ldots$ & $\ldots$ & $\ldots$ \\
\hline K647 & $\ldots$ & $\ldots$ & $\ldots$ & $-116.8 \pm 0.4$ \\
\hline K654 & $\ldots$ & $\ldots$ & $\ldots$ & $-109.5 \pm 0.7$ \\
\hline K672 & $\ldots$ & $\ldots$ & $-106.9 \pm 0.5$ & $\ldots$ \\
\hline K677 & $\ldots$ & $\ldots$ & $-104.9 \pm 0.8$ & $-105.2 \pm 0.9$ \\
\hline K691 & $-109.4 \pm 0.4$ & $-109.6 \pm 0.4$ & $\ldots$ & 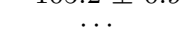 \\
\hline K702 & $-116.7 \pm 0.2$ & $-117.9 \pm 0.3$ & $\ldots$ & $\ldots$ \\
\hline K709 & $\ldots$ & $\ldots$ & $-101.7 \pm 0.4$ & $-99.8 \pm 0.4$ \\
\hline K736 & $\ldots$ & $\ldots$ & $-99.7 \pm 0.4$ & $\ldots$ \\
\hline K757 & $-117.4 \pm 0.3$ & $-111.2 \pm 0.3$ & $\ldots$ & $\ldots$ \\
\hline K800 & $-104.4 \pm 0.8$ & $\ldots$ & $\ldots$ & $\ldots$ \\
\hline K825 & $\cdots$ & $\cdots$ & $-101.4 \pm 0.4$ & $\cdots$ \\
\hline
\end{tabular}


TABLE 5

Radial Velocity of Cluster Members

\begin{tabular}{|c|c|c|c|c|}
\hline K846 & $-105.5 \pm 0.3$ & & $\cdots$ & $-104.6 \pm 0.4$ \\
\hline K853 & $-108.3 \pm 0.3$ & $-109.2 \pm 0.3$ & & $\cdots$ \\
\hline K866 & & & $-109.6 \pm 0.7$ & \\
\hline K875 & $-111.2 \pm 0.4$ & $-110.7 \pm 0.4$ & $\cdots$ & $\cdots$ \\
\hline K879 & $-103.8 \pm 0.4$ & $-104.2 \pm 0.3$ & $\cdots$ & $\cdots$ \\
\hline K902 & $\ldots$ & $\ldots$ & $-108.4 \pm 0.6$ & $-109.0 \pm 0.7$ \\
\hline K906 & $\cdots$ & $\cdots$ & $-106.3 \pm 0.6$ & $\cdots$ \\
\hline K919 & $-111.1 \pm 0.3$ & $-112.4 \pm 0.3$ & $\ldots$ & $\cdots$ \\
\hline K925 & $-108.4 \pm 0.5$ & $-108.1 \pm 0.4$ & $\cdots$ & \\
\hline K926 & $\cdots$ & $\cdots$ & $\cdots$ & $-108.1 \pm 1.0$ \\
\hline K932 & $\cdots$ & $\cdots$ & $\cdots$ & $-107.8 \pm 0.5$ \\
\hline K947 & $\cdots$ & $-116.8 \pm 0.4$ & $\cdots$ & \\
\hline K954 & & $\ldots$ & $\ldots$ & $-104.1 \pm 0.5$ \\
\hline K969 & $-110.8 \pm 0.4$ & $\cdots$ & $\cdots$ & $-108.8 \pm 0.5$ \\
\hline K979 & $-111.2 \pm 0.6$ & $\cdots$ & $\cdots$ & $\ldots$ \\
\hline K989 & & $\cdots$ & $-109.3 \pm 0.6$ & $\cdots$ \\
\hline K993 & $-112.7 \pm 0.3$ & $\cdots$ & $\ldots$ & $\cdots$ \\
\hline K1010 & & $\ldots$ & & $-108.3 \pm 0.8$ \\
\hline K1014 & & . & $-115.4 \pm 0.5$ & $\cdots$ \\
\hline K1029 & $-101.6 \pm 0.4$ & $-102.1 \pm 0.3$ & $\ldots$ & $\cdots$ \\
\hline K1030 & $-100.1 \pm 0.3$ & $-101.1 \pm 0.3$ & $\cdots$ & $\cdots$ \\
\hline K1033 & $-111.4 \pm 0.4$ & $-111.7 \pm 0.3$ & $\cdots$ & $\cdots$ \\
\hline K1040 & & $\cdots$ & $-100.2 \pm 0.3$ & $\cdots$ \\
\hline K1049 & $-106.5 \pm 0.7$ & $\cdots$ & $\ldots$ & $\cdots$ \\
\hline K1054 & $-105.9 \pm 0.4$ & $-106.5 \pm 0.4$ & $\cdots$ & . \\
\hline K1056 & $\cdots$ & . & $\cdots$ & $-103.5 \pm 0.6$ \\
\hline K1069 & $\cdots$ & $\cdots$ & $\cdots$ & $-102.4 \pm 0.4$ \\
\hline K1073 & $-118.8 \pm 0.4$ & $-120.3 \pm 0.4$ & $-119.9 \pm 0.5$ & $-119.9 \pm 0.5$ \\
\hline K1074 & $-106.7 \pm 0.5$ & & $-106.9 \pm 0.6$ & $-107.5 \pm 0.7$ \\
\hline K1079 & $-104.4 \pm 0.4$ & $-106.2 \pm 0.3$ & & $\cdots$ \\
\hline K1083 & $-107.1 \pm 0.6$ & $-106.2 \pm 0.6$ & $-106.5 \pm 0.7$ & $\ldots$ \\
\hline K1084 & $-106.3 \pm 0.4$ & $-105.0 \pm 0.4$ & $-103.7 \pm 0.5$ & \\
\hline K1097 & $-109.9 \pm 0.5$ & $-110.6 \pm 0.5$ & $-108.5 \pm 0.6$ & $-108.8 \pm 0.6$ \\
\hline K1106 & & $-107.1 \pm 0.4$ & $-106.0 \pm 0.5$ & $\cdots$ \\
\hline K1136 & $-103.8 \pm 0.5$ & $-105.3 \pm 0.4$ & $-106.8 \pm 0.5$ & $-105.5 \pm 0.6$ \\
\hline
\end{tabular}

Note. - Data taken on 2006 May 11 and on 2006 October 7 were corrected with a velocity offset of $+1.9 \mathrm{~km} \mathrm{~s}^{-1}$ and $+0.9 \mathrm{~km} \mathrm{~s}^{-1}$ respectively.

a Observations: 1: 2005 May 22, 2: 2006 May 11, 3: 2006 October 4, 4: 2006 October 7. 
TABLE 6

Radial Velocities of Apparent Non-members

\begin{tabular}{|c|c|c|c|c|c|c|c|}
\hline ID No. & $R A(2000)^{\mathrm{a}}$ & $\operatorname{Dec}(2000)^{a}$ & $\mathrm{~V}^{\mathrm{b}}$ & $\mathrm{B}-\mathrm{V}^{\mathrm{b}}$ & $v_{r a d}\left(\mathrm{~km} \mathrm{~s}^{-1}\right)^{\mathrm{c}}$ & $\mathrm{P}^{\mathrm{b}}$ & Obs. ${ }^{d}$ \\
\hline B14 & 212956.70 & +122220.1 & 12.71 & 0.49 & $-22.7 \pm 0.4$ & 99 & $1,2,4$ \\
\hline B22 & 213036.04 & +120517.6 & 13.92 & 0.91 & $-12.1 \pm 0.4$ & 96 & $1,2,3,4$ \\
\hline B25 & 213039.65 & +120523.5 & 12.39 & 1.14 & $-5.4 \pm 0.4$ & 99 & 1,2 \\
\hline C19 & 212952.30 & +115940.2 & 14.89 & 0.87 & $-50.9 \pm 0.6$ & 93 & $1,2,3,4$ \\
\hline K7 & 212927.03 & +120726.9 & 12.83 & 0.88 & $-177.4 \pm 0.5$ & 98 & $1,2,4$ \\
\hline $\mathrm{K} 28$ & 212935.27 & +121440.0 & 13.67 & 1.04 & $-68.5 \pm 0.4$ & 90 & $1,3,4$ \\
\hline K44 & 212958.32 & +120956.5 & 15.36 & 1.04 & $-41.4 \pm 0.5$ & 67 & 3 \\
\hline K73 & 212944.19 & +120917.1 & 13.62 & 0.74 & $-38.4 \pm 0.6$ & 94 & 3 \\
\hline K609 & 212958.84 & +121729.4 & 14.88 & 0.79 & $+4.7 \pm 0.6$ & 96 & $1,2,3,4$ \\
\hline K996 & 213006.80 & +121110.0 & 14.29 & 0.13 & $+15.8 \pm 0.5$ & 99 & 1 \\
\hline K1095 & 213020.32 & +120042.4 & 12.67 & 0.64 & $-1.7 \pm 0.5$ & 99 & $1,2,4$ \\
\hline K1096 & 213020.45 & +121755.9 & 14.03 & 0.60 & $-13.5 \pm 0.5$ & 98 & 1,2 \\
\hline
\end{tabular}

a 2MASS coordinates (Skrutskie et al. 2006).

b The visual photometry and membership probability from proper motions are taken from Cudworth (1976).

c Average radial velocities were calculated from all cross-correlations.

d Observations: 1: 2005 May 22, 2: 2006 May 11, 3: 2006 October 4, 4: 2006 October 7. 
TABLE 7

H $\alpha$ Bisector Velocity of Cluster Members

\begin{tabular}{|c|c|c|c|c|}
\hline ID No. & $\begin{array}{l}v_{b i s, 1} \mathrm{a} \\
\left(\mathrm{km} \mathrm{s}^{-1}\right)\end{array}$ & $\begin{array}{l}v_{b i s, 2}{ }^{\mathrm{a}} \\
\left(\mathrm{km} \mathrm{s}^{-1}\right)\end{array}$ & $\begin{array}{l}v_{b i s, 3}{ }^{\mathrm{a}} \\
\left(\mathrm{km} \mathrm{s}^{-1}\right)\end{array}$ & $\begin{array}{c}v_{b i s, 4}{ }^{\mathrm{a}} \\
\left(\mathrm{km} \mathrm{s}^{-1}\right)\end{array}$ \\
\hline B5 & $-4.2 \pm 1.1$ & .. & $-4.7 \pm 0.7$ & $-3.3 \pm 0.7$ \\
\hline B6 & $-1.8 \pm 0.7$ & $-4.2 \pm 0.7$ & $-6.1 \pm 1.0$ & $-5.5 \pm 1.1$ \\
\hline B16 & & & $-2.7 \pm 0.7$ & \\
\hline B30 & $-4.1 \pm 0.9$ & $-2.5 \pm 1.3$ & $-2.5 \pm 0.4$ & $-2.4 \pm 0.9$ \\
\hline C3 & $-1.8 \pm 1.0$ & $-1.2 \pm 0.7$ & $-0.9 \pm 0.5$ & $+0.3 \pm 0.7$ \\
\hline $\mathrm{C} 20$ & $+0.5 \pm 1.3$ & $-0.4 \pm 1.8$ & $-0.5 \pm 1.0$ & $-0.2 \pm 2.0$ \\
\hline C35 & $-2.2 \pm 0.6$ & $\ldots$ & $\ldots$ & \\
\hline GEB 254 & $\ldots$ & $\ldots$ & $\ldots$ & $-8.5 \pm 0.9$ \\
\hline GEB 289 & $\ldots$ & $\ldots$ & $-3.3 \pm 0.6$ & \\
\hline K12 & $-0.5 \pm 1.0$ & $-1.7 \pm 0.7$ & $-0.6 \pm 0.5$ & $-2.5 \pm 1.2$ \\
\hline K21 & $\cdots$ & $-1.0 \pm 0.8$ & $-0.9 \pm 0.4$ & $-1.7 \pm 0.5$ \\
\hline K22 & $+0.9 \pm 1.1$ & $-1.0 \pm 1.0$ & $-1.8 \pm 0.3$ & $+0.1 \pm 0.6$ \\
\hline K26 & $+0.1 \pm 0.8$ & $+0.2 \pm 0.2$ & $+0.0 \pm 1.5$ & $+0.1 \pm 1.2$ \\
\hline $\mathrm{K} 27$ & $\ldots$ & $+1.2 \pm 0.8$ & $-1.5 \pm 0.8$ & $-1.2 \pm 0.5$ \\
\hline K31 & $\ldots$ & $+0.9 \pm 1.1$ & $-2.3 \pm 0.6$ & $-1.4 \pm 1.3$ \\
\hline K42 & $\cdots$ & & $-0.4 \pm 0.6$ & $+0.7 \pm 0.8$ \\
\hline K47 & $-1.5 \pm 0.7$ & $-0.7 \pm 0.4$ & & $\cdots$ \\
\hline K56 & $-2.2 \pm 0.9$ & $+2.1 \pm 0.7$ & $+0.0 \pm 0.8$ & $-1.7 \pm 0.9$ \\
\hline K60 & & & $+0.4 \pm 0.7$ & $+1.0 \pm 0.4$ \\
\hline K64 & $+0.5 \pm 1.0$ & $+1.7 \pm 1.3$ & $-0.6 \pm 0.5$ & $+0.2 \pm 0.6$ \\
\hline K69 & $-0.6 \pm 0.5$ & $-0.3 \pm 0.3$ & $\ldots$ & $+0.4 \pm 0.7$ \\
\hline K70 & $-0.3 \pm 0.8$ & & & \\
\hline K77 & $-1.8 \pm 0.6$ & $-1.8 \pm 0.6$ & $-2.1 \pm 0.6$ & $-2.2 \pm 0.5$ \\
\hline K87 & $\cdots$ & & & $-3.9 \pm 1.3$ \\
\hline K89 & $\ldots$ & $-0.7 \pm 0.7$ & $-1.6 \pm 0.5$ & $-0.8 \pm 0.5$ \\
\hline K92 & $-1.7 \pm 1.2$ & $+1.1 \pm 0.5$ & $+0.5 \pm 0.7$ & $-1.0 \pm 1.2$ \\
\hline K105 & $-2.7 \pm 1.5$ & $\ldots$ & 1 & $-2.0 \pm 1.2$ \\
\hline K112 & $\ldots$ & $\ldots$ & & $+0.9 \pm 1.6$ \\
\hline K114 & $\ldots$ & $\cdots$ & $-1.7 \pm 0.4$ & $\ldots$ \\
\hline K129 & $\cdots$ & $\cdots$ & $+0.1 \pm 0.4$ & $\cdots$ \\
\hline K133 & $\ldots$ & $-0.8 \pm 0.6$ & $+0.5 \pm 1.6$ & \\
\hline K136 & $\cdots$ & $\ldots$ & & $-2.9 \pm 0.8$ \\
\hline K137 & $\ldots$ & $\ldots$ & $+1.5 \pm 1.3$ & $\ldots$ \\
\hline K144 & $\cdots$ & $-5.3 \pm 1.3$ & & $-5.7 \pm 0.9$ \\
\hline K145 & $+0.3 \pm 0.9$ & $-0.2 \pm 0.6$ & $-0.4 \pm 1.2$ & $\ldots$ \\
\hline $\mathrm{K} 146$ & $\ldots$ & $-2.5 \pm 0.6$ & & $\cdots$ \\
\hline K151 & $\cdots$ & $\ldots$ & $+0.9 \pm 0.8$ & $\ldots$ \\
\hline K152 & $-0.4 \pm 1.0$ & $-0.9 \pm 0.8$ & $+0.2 \pm 1.1$ & $\ldots$ \\
\hline K153 & $+0.0 \pm 1.0$ & - & $+0.7 \pm 1.2$ & $-0.4 \pm 1.5$ \\
\hline K158 & $\cdots$ & $-11.1 \pm 1.0$ & & $\ldots$ \\
\hline K202 & $\cdots$ & & $+0.1 \pm 0.8$ & $\cdots$ \\
\hline K224 & $-2.9 \pm 0.4$ & $-4.0 \pm 0.6$ & $-4.5 \pm 0.9$ & $\cdots$ \\
\hline $\mathrm{K} 238$ & $\cdots$ & & $\ldots$ & $-3.1 \pm 0.6$ \\
\hline K255 & $-1.1 \pm 1.8$ & $-1.0 \pm 0.5$ & $\cdots$ & $\ldots$ \\
\hline K260 & $-3.0 \pm 1.2$ & $-10.6 \pm 0.9$ & $\ldots$ & $\ldots$ \\
\hline $\mathrm{K} 272$ & $\ldots$ & $\ldots$ & $-3.0 \pm 1.6$ & $\ldots$ \\
\hline $\mathrm{K} 288$ & $-0.91 \pm 0.82$ & $\ldots$ & $-2.7 \pm 0.6$ & $\ldots$ \\
\hline K328 & $\ldots$ & $-2.4 \pm 0.6$ & & $-3.3 \pm 1.3$ \\
\hline K337 & $\cdots$ & $+0.1 \pm 0.6$ & $-0.4 \pm 1.2$ & $+2.9 \pm 1.6$ \\
\hline K341 & $-3.2 \pm 0.6$ & $-6.9 \pm 1.0$ & $-6.2 \pm 0.6$ & $-6.3 \pm 0.9$ \\
\hline K361 & $\ldots$ & $\ldots$ & $-1.9 \pm 0.4$ & $\ldots$ \\
\hline K393 & $-2.0 \pm 0.9$ & $\ldots$ & $\ldots$ & $\ldots$ \\
\hline $\mathrm{K} 421$ & $\ldots$ & $\ldots$ & $-4.3 \pm 0.7$ & $\ldots$ \\
\hline $\mathrm{K} 431$ & $-4.2 \pm 0.8$ & $-5.0 \pm 1.3$ & $-5.7 \pm 1.1$ & $\cdots$ \\
\hline K447 & $\cdots$ & $-2.0 \pm 0.6$ & $\ldots$ & $\ldots$ \\
\hline K462 & $\cdots$ & $-3.6 \pm 0.7$ & $\cdots$ & $\ldots$ \\
\hline K476 & $\ldots$ & $-0.2 \pm 0.5$ & $\cdots$ & $\ldots$ \\
\hline K479 & $\ldots$ & $\ldots$ & $\ldots$ & $-0.7 \pm 0.7$ \\
\hline K482 & $\ldots$ & $\ldots$ & $-6.2 \pm 1.2$ & $\ldots$ \\
\hline K506 & $\ldots$ & $\ldots$ & $\ldots$ & $-0.6 \pm 1.9$ \\
\hline K550 & $\cdots$ & $-1.3 \pm 1.1$ & $+1.6 \pm 0.4$ & $+1.6 \pm 0.6$ \\
\hline K567 & $\cdots$ & $\ldots$ & $-5.7 \pm 1.1$ & $\ldots$ \\
\hline K582 & $-13.2 \pm 1.6$ & $\cdots$ & $\cdots$ & $\cdots$ \\
\hline K583 & $-6.0 \pm 1.3$ & $\ldots$ & $\ldots$ & $\ldots$ \\
\hline K647 & $\ldots$ & $\ldots$ & $\ldots$ & $-6.2 \pm 0.8$ \\
\hline K654 & $\ldots$ & $\ldots$ & $\ldots$ & $+0.1 \pm 0.7$ \\
\hline K672 & $\cdots$ & $\cdots$ & $-4.6 \pm 0.9$ & \\
\hline K677 & $\cdots$ & $\ldots$ & $-1.1 \pm 1.5$ & $-4.6 \pm 1.9$ \\
\hline K691 & $-1.2 \pm 0.5$ & $-0.6 \pm 0.5$ & $\ldots$ & $\ldots$ \\
\hline K702 & $-6.1 \pm 0.8$ & $-3.9 \pm 0.9$ & $\ldots$ & \\
\hline K709 & $\ldots$ & $\ldots$ & $-3.1 \pm 0.6$ & $-4.2 \pm 1.0$ \\
\hline K736 & $\ldots$ & $\ldots$ & $-2.1 \pm 0.3$ & $\ldots$ \\
\hline K757 & $-2.8 \pm 0.5$ & $-8.9 \pm 1.1$ & & $\cdots$ \\
\hline K800 & $+1.6 \pm 0.6$ & $\cdots$ & $\cdots$ & $\cdots$ \\
\hline K825 & $\ldots$ & $\cdots$ & $+0.2 \pm 0.9$ & $\ldots$ \\
\hline
\end{tabular}


TABLE 7

H $\alpha$ Bisector Velocity of Cluster Members

\begin{tabular}{|c|c|c|c|c|}
\hline K846 & $-1.8 \pm 0.5$ & & & $-3.3 \pm 1.2$ \\
\hline K853 & $-1.8 \pm 0.5$ & $-2.2 \pm 0.8$ & & $\cdots$ \\
\hline K866 & & & $-1.3 \pm 0.4$ & $\cdots$ \\
\hline K875 & $-7.2 \pm 1.0$ & $-4.4 \pm 0.4$ & 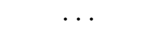 & $\ldots$ \\
\hline 879 & $-1.3 \pm 0.5$ & $-0.5 \pm 0.6$ & & \\
\hline K902 & $\ldots$ & $\cdots$ & $-0.4 \pm 0.4$ & $+0.8 \pm 0.5$ \\
\hline K906 & $\cdots$ & $\cdots$ & $-0.9 \pm 0.4$ & $\ldots$ \\
\hline K919 & $-2.4 \pm 0.5$ & $-2.6 \pm 0.8$ & $\ldots$ & $\ldots$ \\
\hline 925 & $-0.7 \pm 0.7$ & $-0.4 \pm 0.8$ & $\ldots$ & $\ldots$ \\
\hline K926 & $\ldots$ & $\ldots$ & $\cdots$ & $+0.6 \pm 0.7$ \\
\hline K932 & $\cdots$ & & $\ldots$ & $-1.5 \pm 1.2$ \\
\hline K947 & $\cdots$ & $-1.0 \pm 1.1$ & $\cdots$ & $\cdots$ \\
\hline K954 & $\ldots$ & $\ldots$ & $\ldots$ & $-0.6 \pm 0.8$ \\
\hline K969 & $-4.0 \pm 0.6$ & $\ldots$ & $\ldots$ & $-1.7 \pm 0.3$ \\
\hline K979 & $-6.7 \pm 1.3$ & $\ldots$ & $\ldots$ & $\ldots$ \\
\hline K989 & & $\ldots$ & $-0.4 \pm 1.0$ & $\ldots$ \\
\hline K993 & $-2.4 \pm 0.9$ & $\ldots$ & $\ldots$ & $\ldots$ \\
\hline K1010 & $\cdots$ & $\ldots$ & $\ldots$ & $-2.3 \pm 1.6$ \\
\hline K1014 & $\cdots$ & $\cdots$ & $+1.1 \pm 0.9$ & $\ldots$ \\
\hline K1029 & $-2.9 \pm 1.1$ & $-3.0 \pm 1.0$ & $\ldots$ & $\ldots$ \\
\hline K1030 & $-1.3 \pm 0.5$ & $-2.6 \pm 0.6$ & $\cdots$ & $\cdots$ \\
\hline K1033 & $-0.9 \pm 0.5$ & $+0.6 \pm 0.7$ & $\cdots$ & $\cdots$ \\
\hline K1040 & & & $-6.3 \pm 1.4$ & $\cdots$ \\
\hline K1049 & $-1.2 \pm 1.8$ & & & $\cdots$ \\
\hline K1054 & $-1.5 \pm 0.4$ & $-1.1 \pm 0.6$ & $\cdots$ & $\cdots$ \\
\hline K1056 & $\cdots$ & $\ldots$ & $\cdots$ & $-0.4 \pm 1.0$ \\
\hline K1069 & $\ldots$ & & & $-1.5 \pm 0.8$ \\
\hline K1073 & $-3.6 \pm 0.8$ & $-0.7 \pm 0.7$ & $+0.0 \pm 0.8$ & $-1.9 \pm 0.9$ \\
\hline K1074 & $-0.1 \pm 0.9$ & & $-0.5 \pm 1.6$ & $-0.6 \pm 1.1$ \\
\hline K1079 & $-1.4 \pm 0.4$ & $-0.9 \pm 0.7$ & & $\ldots$ \\
\hline K1083 & $-0.8 \pm 1.6$ & $-1.6 \pm 1.6$ & $+1.5 \pm 1.2$ & $\cdots$ \\
\hline K1084 & $-0.7 \pm 0.8$ & $-0.6 \pm 0.6$ & $-1.1 \pm 0.5$ & $\cdots$ \\
\hline K1097 & $+2.5 \pm 0.8$ & $+0.6 \pm 0.8$ & $-1.0 \pm 0.7$ & $-1.1 \pm 0.6$ \\
\hline K1106 & & $-0.2 \pm 0.7$ & $-1.7 \pm 1.5$ & \\
\hline K1136 & $-1.0 \pm 1.4$ & $-0.2 \pm 0.7$ & $+0.9 \pm 0.7$ & $-1.3 \pm 1.0$ \\
\hline
\end{tabular}

a Observations: 1: 2005 May 22; 2: 2006 May 11; 3: 2006 October 4; 4: 2006 October 7 . 
TABLE 8

Parameters of Ca it K Line for Stars Showing Emission

\begin{tabular}{|c|c|}
\hline ID No. & $B / R 2005$ May 23 \\
\hline K77 & $>1$ \\
\hline K224 & $<1$ \\
\hline K260 & $>1$ \\
\hline K341 & $>1$ \\
\hline K393 & $>1$ \\
\hline K582 & $<1$ \\
\hline K702 & not clear \\
\hline K757 & $<1$ \\
\hline K853 & $>1$ \\
\hline K875 & 1 \\
\hline K879 & $>1$ \\
\hline K969 & 1 \\
\hline K979 & not clear \\
\hline K1029 & not clear \\
\hline
\end{tabular}

Note. - The parameter $B / R$ is the intensity ratio of Blue (short wavelength) and Red (long wavelength) emission peaks. 